\title{
Solução Numérica de Escoamentos Axisimétricos Não-Newtonianos com Superfícies Livres
}

\author{
LUCIANE GRossi
}

Orientador: Prof. Dr. Murilo F. TOMÉ

Dissertação apresentada ao Instituto de Ciências Matemáticas de São Carlos, da Universidade de São Paulo, como parte dos requisitos para obtenção do título de Mestre em Ciências - Área: Ciência de Computação e Matemática Computacional.

São Carlos - SP

1997 
À DEUS por tudo permitir e possibilitar. À minha família. 


\section{Agradecimentos}

Ao meu orientador Prof. Dr. Murilo Francisco Tomé que mesmo distante soube orientar-me com afinco no decorrer deste trabalho. Obrigada pelo apoio, incentivo, dedicação e amizade.

Em especial ao professor Antônio Castelo Filho pela dedicação e paciência que atendeume em várias dúvidas surgidas na parte técnica e operacional. A você minha sincera gratidão.

Aos professores José Alberto Cuminato e Armando Oliveira Fortuna pelas valiosas sugestões e pelo incentivo.

Aos professores do Departamento de Matemática da UFMT, em especial aos professores Carlos R. Sanches, Olga Nakajima e aos professores do GEPEMAT que incentivaramme no ingresso do programa de mestrado.

As secretárias Beth, Laura e Marília pela atenção, as bibliotecárias pela disponibilidade e a todos os funcionários de maneira geral.

As minhas grandes amigas de Cuiabá: Cidinha e Celminha, pela amizade sempre presente.

A minha família que tanto amo, pelo incentivo e pelas valiosas orações.

Aos amigos Cidinho e Mari pelo incentivo a vir para São Carlos, são eles de certa forma os "culpados" responsáveis por este momento.

Aos amigos que conheci em São Carlos, que tornaram estes anos mais agradáveis; em especial ao Lauro, presente em muitas madrugadas de trabalho, sempre prestativo e amigo; também ao Sadao pelas muitas vezes que me socorreu nas dúvidas.

Em especial a turminha da sala 138 com os quais tive o privilégio de compartilhar muitos risos e muito trabalho; aos amigos da sala 133, 141 e 145.

Ao meu namorado Marcos Bombacini pelo carinho, apoio, incentivo e compreensão.

Agradeço ao CNPQ pelo suporte financeiro.

À DEUS, pelo dom da vida e por mais essa realização.

Finalmente, agradeço a todos que direta ou indiretamente me ajudaram na execução deste trabalho. 


\section{Resumo}

Este trabalho apresenta um método numérico para resolver escoamentos axisimétricos não-Newtonianos com superfícies livres. A metodologia empregada é uma extensão do código bidimensional GENSMAC para problemas axisimétricos. O código GENSMAC é uma técnica numérica que utiliza o método das particulas marcadoras ("marker-andcell") para simular escoamentos incompressíveis transientes. As equações governantes são resolvidas usando o método de diferenças finitas numa malha diferenciada ("staggered grid"). O fluido é representado por partículas marcadoras, as quais permitem a localização e visualização da superfície livre do fluido. Vários exemplos que demonstram a aplicação dessa nova técnica são apresentados. Em particular, a simulação de enchimento de moldes, do "die-swell" e do "splashing drop" são apresentados. 


\begin{abstract}
This work presents a numerical method for solving axisymmetric non-Newtonian flows. The methodology is an extension of the two-dimensional GENSMAC code to axisymmetric flows. GENSMAC is a marker-and-cell technique for investigating the dynamics of an incompressible fluid. It solves the governing equations using the finite difference method on a staggered grid. The fluid is represented by marker particles which provide the location and visualization of the free surface. Various results demonstrating the applicability of this new technique is presented. In particular, the simulation of die-swell, container filling and the splashing drop is given.
\end{abstract}




\section{Sumário}

1 Introdução 1

1.1 Introdução . . . . . . . . . . . . . . . . . . . . . . . 1

1.2 Organização da Dissertação . . . . . . . . . . . . . . . . . . . 2

2 Equações Governantes para Escoamentos Axisimétricos não-Newtonianos 4

2.1 Equação de Momento . . . . . . . . . . . . . . . . . . . . . . . . 4

2.2 Equação da Shear Rate . . . . . . . . . . . . . . . . . . . . . 7

2.3 Condições de Contorno na Superfície Livre . . . . . . . . . . . . . . . 8

3 Código GENSMAC 10

3.1 Características do Código . . . . . . . . . . . . . . . . . 10

3.2 Algoritmo Computacional . . . . . . . . . . . . . . . . . . 11

3.3 Cálculo da Shear Rate na Fronteira Rígida . . . . . . . . . . . . . . . . . 14

4 Aproximação por Diferenças Finitas 16

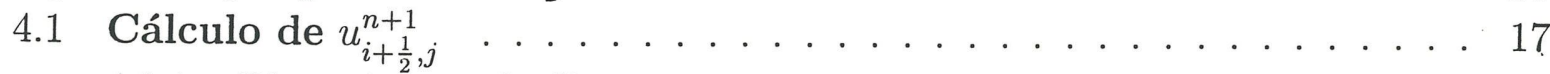

4.1.1 Discretização da Shear Rate . . . . . . . . . . . . . . . . . . 18

4.1 .2 Cálculo da Viscosidade . . . . . . . . . . . . . . . . 20

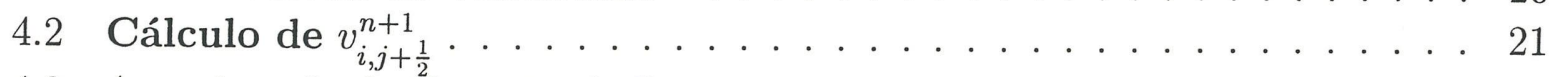

4.3 Aproximação da Equação de Poisson . . . . . . . . . . . . . . . . . . . 21

4.4 Definição das Células . . . . . . . . . . . . . . . . . . . . . . . 22

4.5 Aproximação das Condições de Contorno na Superfície Livre . . . . . . . . 23

4.6 Cálculo do Time-Step . . . . . . . . . . . . . . . . . . . . . . . . . 28

4.7 A Solução da Equação de Poisson . . . . . . . . . . . . . . . . . . . 28

4.7.1 Implementação do Método dos Gradientes Conjugados . . . . . . . 29

4.8 Movimento das Partículas . . . . . . . . . . . . . . . . . . . . 29

5 Exemplos Numéricos e Aplicações 30

5.1 Alterações Realizadas no Código GENSMAC . . . . . . . . . . . . . . . . 30

5.2 Exemplos Numéricos e Aplicações . . . . . . . . . . . . . . . . . . . . 31

5.2.1 Simulação Numérica do Enchimento de Cavidades . . . . . . . . . . 31

5.2.2 Simulação Numérica do "Die Swell" de um Jato Axisimétrico . . . . 36 
5.2.3 Simulação Numérica do Splashing Drop . . . . . . . . . . . . . . . 38

6 Resumo e Conclusões $\quad 44$ 


\section{Capítulo 1}

\section{Introdução}

\subsection{Introdução}

Devido ao grande avanço computacional, tecnológico e ao surgimento de eficientes técnicas computacionais, tornou-se possível a solução numérica de vários problemas de mecânica dos fluidos. A experimentação em laboratório é de altíssimo custo e muitas vezes surgem grandes dificuldades em reproduzir as condições físicas e ainda consomem muito tempo. O uso de técnicas numéricas têm-se mostrado ser o melhor caminho atualmente, praticamente não apresenta restrições, podendo resolver problemas com condições de contorno complicadas, definidas em geometrias complexas e apresentando resultados com grande rapidez. Entre os diversos problemas surgidos na mecânica dos fluidos, a classe de problemas chamados "escoamentos com superfícies livres" têm atraído a atenção de muitos pesquisadores. Estamos constantemente em contato com este tipo de escoamento, como por exemplo a água escoando de uma torneira, o mel escoando de uma garrafa, o escoamento do creme dental ao comprimirmos o frasco, o gotejar de um remédio em ùm copo d'agua, etc. Este tipo de problema também aparece em muitas aplicações industriais tal como a injeção de material em moldes como por exemplo: injeção de plástico, ligas metálicas e fluidos utilizados na indústria alimentícia. Existe portanto um grande interesse no desenvolvimento de técnicas numéricas para resolver esta classe de problemas e prever como o escoamento se comporta durante o processo industrial (onde a simulação desempenha papel decisivo nos custos e qualidade do projeto). Porém, esta não é uma tarefa fácil, já que nestes problemas a superfície livre muda continuamente sua posição no tempo tomando diferentes formas. Outra dificuldade surge do tipo de condições de contorno as quais devem ser satisfeitas na superfície livre cujo formato é desconhecido e ainda do fato de que muitos problemas envolvem geometrias altamente complexas.

Contudo o desenvolvimento de técnicas numéricas para simular escoamentos com superfícies livres tem sido uma área de intensa pesquisa nas últimas décadas. Em particular, o método MAC (Marker-and-Cell) [1] tem sido desenvolvido por muitos pesquisadores. Entre muitas das modificações feitas no método MAC podemos citar o método SMAC (Simplified-Marker-and-Cell) [2] e o código GENSMAC (Generalized-Simplified-Markerand-Cell) introduzido por Tomé and McKee [7]. GENSMAC é uma extensão do método 
SMAC voltado para o cálculo de escoamentos transientes bidimensionais com superfícies livres em um domínio arbitrário com condições de contorno do tipo "no-slip" e "free-slip" e vários "inflows" e "outflows" podem ser especificados. O código utiliza uma técnica de diferenças finitas para resolver escoamentos incompressíveis numa malha diferenciada empregando a velocidade e pressão como variáveis dependentes. Uma característica do método é o uso de partículas marcadoras que permitem a visualização do escoamento bem como a aplicação das condições de contorno na superfície livre do fluido. Recentemente, Tomé et al. [8] extenderam o código GENSMAC para o tratamento de escoamentos bidimensionais não-Newtonianos para fluidos do tipo "power-law" e "Cross model". Embora GENSMAC possa ser aplicado a muitos problemas de engenharia, o código ainda não possui uma facilidade para simular escoamentos axisimétricos não-Newtonianos. Com o objetivo de ampliar a gama de aplicabilidade do GENSMAC e torná-lo mais geral, esse trabalho apresenta uma extensão da técnica introduzida por Tomé et al. [8] para o tratamento de escoamentos axisimétricos não-Newtonianos com superfícies livres.

Neste trabalho apresentamos um estudo das equações governantes para escoamentos incompressíveis axisimétricos com superfícies livres para um fluido generalizado do tipo "Cross Model" e "Power-Law". Também são considerados a discretização dessas equações e a implementação no método apresentado no código GENSMAC [8] usando coordenadas cilíndricas e a apresentação de resultados numéricos.

\subsection{Organização da Dissertação}

Esta dissertação divide-se em 6 capítulos.

- No Capítulo 1 é apresentada uma introdução geral com um breve histórico sobre as técnicas de diferenças finitas utilizadas para simular problemas de escoamentos com superfícies livres.

- O Capítulo 2 apresenta as equações governantes para escoamento de fluidos nãoNewtonianos com superfícies livres juntamente com um detalhado desenvolvimento em coordenadas cilíndricas das equações básicas apresentadas.

- No Capítulo 3 ilustramos algumas características do código Gensmac e apresentamos um método numérico para resolver escoamentos axisimétricos não-Newtonianos com superfícies livres.

- No Capítulo 4, utilizamos a técnica de diferenças finitas para obter uma solução aproximada das equações desenvolvidas no capítulo 2 e para a equação de Poisson.

- No Capítulo 5 são apresentadas as alterações necessárias no código GENSMAC que possibilitaram a inclusão de coordenadas cilíndricas no código. Também, são 
apresentados vários exemplos numéricos demonstrando a aplicabilidade dessa nova técnica para resolver escoamentos axisimétricos não-Newtonianos.

- O capítulo 6 constitui-se da conclusão e das perspectivas de trabalho futuro . 


\section{Capítulo 2}

\section{Equações Governantes para Escoamentos Axisimétricos não-Newtonianos}

Este capítulo apresenta as equações fundamentais para o escoamento de fluídos nãoNewtonianos e o desenvolvimento detalhado destas equações em coordenadas cilíndricas.

\subsection{Equação de Momento}

As equações básicas para escoamentos viscosos incompressíveis dependentes do tempo, são as equações de momento juntamente com a condição de incompressibilidade do fluido de densidade $\rho$ que se apresenta da seguinte forma:

$$
\begin{gathered}
\rho \frac{D \mathbf{u}}{D t}=\nabla \cdot \underline{\underline{\sigma}}+\rho \mathbf{g}, \\
\nabla \cdot \mathbf{u}=0 .
\end{gathered}
$$

onde $\mathbf{u}=\mathbf{u}(r, z, t), \underline{\underline{\sigma}}$ e $\mathbf{g}$ denotam a velocidade, o tensor tensão e a aceleração gravitacional do fluido, respectivamente. Como pretendemos tratar de escoamentos nãoNewtonianos de um fluido generalizado, serão consideradas as seguintes equações constitutivas:

$$
\begin{gathered}
\underline{\underline{\boldsymbol{\sigma}}}=-p \underline{\underline{\mathbf{I}}}+\underline{\underline{\tau}}, \quad \underline{\underline{\tau}}=2 \mu(q) \underline{\underline{\mathbf{d}}}, \\
\underline{\underline{\mathbf{d}}}=\frac{1}{2}\left[(\nabla \mathbf{u})+(\nabla \mathbf{u})^{T}\right]
\end{gathered}
$$

sendo que $p, \underline{\underline{\mathbf{I}}}, \underline{\underline{\tau}}$ e $\underline{\underline{\mathbf{d}}}$ denotam a pressão, o tensor identidade, o tensor extra tensão e o tensor razão de deformação de um fluido, respectivamente. q é a razão de cisalhamento local definida por

$$
q=\left(2 \operatorname{tr}\left(\underline{\underline{\mathbf{d}}}^{2}\right)\right)^{\frac{1}{2}}
$$


e $\mu(q)$ é a viscosidade aparente (uma função conhecida de $q$ ) e $\nu=\mu / \rho$ a viscosidade cinemática.

Substituindo as equações (2.3) e (2.4) na equação (2.1), a equação de momento pode ser expressa na seguinte forma vetorial:

$$
\rho \frac{D \mathbf{u}}{D t}=-\nabla p+\mu(q) \nabla^{2} \mathbf{u}+(\nabla \mu(q)) \cdot\left[(\nabla \mathbf{u})+(\nabla \mathbf{u})^{T}\right]+\rho \mathbf{g} .
$$

Desenvolvendo o termo $\frac{D \mathbf{u}}{D t}$ obtem-se:

e aplicando-o sobre o vetor $\mathbf{u}$ fornece:

$$
\frac{D}{D t}=\frac{\partial}{\partial t}+(\mathbf{u} \cdot \nabla)
$$

$$
\frac{D \mathbf{u}}{D t}=\frac{\partial \mathbf{u}}{\partial t}+(\mathbf{u} \cdot \nabla) \mathbf{u}
$$

que na forma conservativa é dado por

$$
\frac{D \mathbf{u}}{D t}=\frac{\partial \mathbf{u}}{\partial t}+\nabla \cdot(\mathbf{u u})
$$

Considerando escoamentos axisimétricos $(\partial / \partial \theta=0)$ e usando coordenadas cilíndricas Orz, com $\mathbf{u}=u(r, z, t) \mathbf{e}_{\mathbf{r}}+v(r, z, t) \mathbf{e}_{\mathbf{z}}$ e lembrando que o divergente em coordenadas cilíndricas é $\nabla \cdot \mathbf{F}=\frac{1}{r} \frac{\partial\left(r \cdot F_{r}\right)}{\partial r}+\frac{1}{r} \frac{\partial F_{v}}{\partial \theta}+\frac{\partial F_{z}}{\partial z}$ (ver Acheson [10], pag. 352), temos que

$$
\frac{D \mathbf{u}}{D t}=\left[\begin{array}{l}
\frac{\partial u}{\partial t}+\frac{1}{r} \frac{\partial\left(r u^{2}\right)}{\partial r}+\frac{\partial(v u)}{\delta z} \\
\frac{\partial v}{\partial t}+\frac{1}{r} \frac{\partial(r u v)}{\partial r}+\frac{\partial\left(v^{2}\right)}{\delta z}
\end{array}\right] .
$$

Para desenvolver o termo $\nabla^{2} \mathbf{u}$, faremos uso da identidade vetorial (ver [10]. pág. 348)

$$
\nabla^{2} \mathbf{u}=\nabla(\nabla \cdot \mathbf{u})-\nabla \wedge(\nabla \wedge \mathbf{u})
$$

e usando a condição (2.2), obtemos

$$
\nabla^{2} \mathbf{u}=-\nabla \wedge(\nabla \wedge \mathbf{u})
$$

que escrito em coordenadas cilíndricas produz:

$$
\nabla^{2} \mathbf{u}=\frac{\partial}{\partial z}\left(\frac{\partial u}{\partial z}-\frac{\partial v}{\partial r}\right) \mathbf{e}_{\mathbf{r}}-\frac{1}{r} \frac{\partial}{\partial r}\left(r\left(\frac{\partial u}{\partial z}-\frac{\partial v}{\partial r}\right)\right) \mathbf{e}_{\mathbf{z}}
$$

Agora trabalhando o termo $(\nabla \mu(q)) \cdot\left[(\nabla \mathbf{u})+(\nabla \mathbf{u})^{T}\right]$, (conforme [10]), temos

$$
\nabla \mathbf{u}=\left[\begin{array}{ccc}
\frac{\partial u}{\partial r} & 0 & \frac{\partial u}{\partial z} \\
0 & \frac{u}{r} & 0 \\
\frac{\partial v}{\partial r} & 0 & \frac{\partial v}{\partial z}
\end{array}\right]
$$


donde podemos escrever (visto que $\nabla \mu(q)=\frac{\partial \mu(q)}{\partial r} \mathbf{e}_{\mathbf{r}}+\frac{\partial \mu(q)}{\partial z} \mathbf{e}_{\mathbf{z}}$ )

$$
(\nabla \mu(q)) \cdot\left[(\nabla \mathbf{u})+(\nabla \mathbf{u})^{T}\right]=\left[\begin{array}{cc}
2 \frac{\partial u}{\partial r} \frac{\partial \mu(q)}{\partial r}+\left(\frac{\partial u}{\partial z}+\frac{\partial v}{\partial r}\right) \frac{\partial \mu(q)}{\delta z} \\
\left(\frac{\partial u}{\partial z}+\frac{\partial v}{\partial r}\right)^{\cdot / \partial \mu(q)} \frac{\partial p}{\partial r}+ \\
2 \frac{\partial v}{\delta z} \frac{\mu(q)}{\partial z}
\end{array}\right]
$$

Substituindo as equações (2.7), (2.8) e (2.10) em (2.6), a equação de momento toma a forma:

$$
\begin{gathered}
\frac{\partial u}{\partial t}+\frac{1}{r} \frac{\partial\left(r u^{2}\right)}{\partial r}+\frac{\partial(u v)}{\partial z}=-\frac{1}{\rho} \frac{\partial p}{\partial r}+\nu(q) \frac{\partial}{\partial z}\left(\frac{\partial u}{\partial z}-\frac{\partial v}{\partial r}\right) \\
+2 \frac{\partial u}{\partial r} \frac{\partial \nu(q)}{\partial r}+\left(\frac{\partial u}{\partial z}+\frac{\partial v}{\partial r}\right) \frac{\partial \nu(q)}{\partial z}+g_{r} \\
\frac{\partial v}{\partial t}+\frac{1}{r} \frac{\partial(r u v)}{\partial r} \quad+\frac{\partial\left(v^{2}\right)}{\partial z}=-\frac{1}{\rho} \frac{\partial p}{\partial z}-\nu(q) \frac{1}{r} \frac{\partial}{\partial z}\left(r\left(\frac{\partial u}{\partial z}-\frac{\partial v}{\partial r}\right)\right) \\
+\left(\frac{\partial u}{\partial z}+\frac{\partial v}{\partial r}\right) \frac{\partial \nu(q)}{\partial r}+2 \frac{\partial u}{\partial z} \frac{\partial \nu(q)}{\partial z}+g_{z}
\end{gathered}
$$

onde $\mathbf{g}=g_{r} \mathbf{e}_{\mathbf{r}}+g_{z} \mathbf{e}_{\mathbf{z}}$.

Introduzindo a seguir uma adimensionalização nas equações acima, com $L, U$ e $\nu_{0}$ denotando unidades de comprimento, velocidade e viscosidade, tem-se:

$u=U \bar{u}, \quad v=U \bar{v}, \quad r=L \bar{r}$,

$z=L \bar{z}, \quad \nu=\nu_{0} \bar{\nu}, \quad q=\frac{U}{L} \bar{q}$

$t=\frac{L}{U} \bar{t}, \quad p=\rho U^{2} \bar{p}, \quad \mathbf{g}=g \overline{\mathbf{g}}$.

onde $g=|\mathbf{g}|$, ( $\overline{\mathbf{g}}$ é um vetor unitário).

Logo, as equações (2.11), (2.12) e (2.2) na forma adimensionalizada podem ser reescritas como:

$$
\begin{aligned}
\frac{\partial u}{\partial t}= & -\frac{1}{r} \frac{\partial\left(r u^{2}\right)}{\partial r}-\frac{\partial(u v)}{\partial z}-\frac{\partial p}{\partial r}+\frac{\nu(q)}{R e} \frac{\partial}{\partial z}\left(\frac{\partial u}{\partial z}-\frac{\partial v}{\partial r}\right) \\
& +\frac{1}{R e}\left[2 \frac{\partial u}{\partial r} \frac{\partial \nu}{\partial r}+\left(\frac{\partial u}{\partial z}+\frac{\partial v}{\partial r}\right) \frac{\partial \nu}{\partial z}\right]+\frac{1}{F r^{2}} g_{r}, \\
\frac{\partial v}{\partial t}=- & -\frac{1}{r} \frac{\partial(r u v)}{\partial r}-\frac{\partial\left(v^{2}\right)}{\partial z}-\frac{\partial p}{\partial z}-\frac{\nu(q)}{R e} \frac{1}{r} \frac{\partial}{\partial z}\left(r\left(\frac{\partial u}{\partial z}-\frac{\partial v}{\partial r}\right)\right) \\
+ & \frac{1}{R e}\left[\left(\frac{\partial u}{\partial z}+\frac{\partial v}{\partial r}\right) \frac{\partial \nu}{\partial r}+2 \frac{\partial u}{\partial z} \frac{\partial \nu}{\partial z}\right]+\frac{1}{F r^{2}} g_{z},
\end{aligned}
$$




$$
\frac{1}{r} \frac{\partial(r u)}{\partial r}+\frac{\partial v}{\partial z}=0
$$

Nas equações acima, $R e=\frac{U L}{\nu_{0}}$ e $F r=\frac{U}{\sqrt{L g}}$ denotam os números de Reynolds e Froude, respectivamente.

No Capítulo a seguir, faremos uso das equações de momento (2.13) e (2.14) escritas na forma:

$$
\frac{\partial \mathbf{u}}{\partial t}=-\nabla p+\mathbf{N}(\mathbf{u})
$$

onde $\mathbf{N}(\mathbf{u})$ tem como componentes

$$
\begin{aligned}
N_{1}= & -\frac{1}{r} \frac{\partial\left(r u^{2}\right)}{\partial r}-\frac{\partial(u v)}{\partial z}+\frac{\nu(q)}{R e} \frac{\partial}{\partial z}\left(\frac{\partial u}{\partial z}-\frac{\partial v}{\partial r}\right) \\
& +\frac{1}{R e}\left[2 \frac{\partial u}{\partial r} \frac{\partial \nu}{\partial r}+\left(\frac{\partial u}{\partial z}+\frac{\partial v}{\partial r}\right) \frac{\partial \nu}{\partial z}\right]+\frac{1}{F r^{2}} g_{r}, \\
N_{2}=- & -\frac{1}{r} \frac{\partial(r u v)}{\partial r}-\frac{\partial\left(v^{2}\right)}{\partial z}-\frac{\nu(q)}{R e} \frac{1}{r} \frac{\partial}{\partial z}\left(r\left(\frac{\partial u}{\partial z}-\frac{\partial v}{\partial r}\right)\right) \\
+ & \frac{1}{R e}\left[\left(\frac{\partial u}{\partial z}+\frac{\partial v}{\partial r}\right) \frac{\partial \nu}{\partial r}+2 \frac{\partial u}{\partial z} \frac{\partial \nu}{\partial z}\right]+\frac{1}{F r^{2}} g_{z} .
\end{aligned}
$$

\subsection{Equação da Shear Rate}

A razão de cisalhamento é definida pela fórmula (conforme (2.9)):

$$
q=\left(2 \operatorname{tr}\left(\underline{\underline{\mathrm{d}}}^{2}\right)\right)^{\frac{1}{2}}
$$

onde $\quad \underline{\underline{\mathbf{d}}}=\frac{1}{2}\left[(\nabla \mathbf{u})+(\nabla \mathbf{u})^{T}\right]$.

Escrevendo a equação (2.19) em coordenadas cilíndricas, temos:

$$
q=\left[\operatorname{tr}\left(\frac{1}{4}\left[\begin{array}{ccc}
2 \frac{\partial u}{\partial r} & 0 & \frac{\partial u}{\partial z}+\frac{\partial v}{\partial r} \\
0 & 2 \frac{u}{r} & 0 \\
\frac{\partial u}{\partial z}+\frac{\partial v}{\partial r} & 0 & 2 \frac{\partial v}{\partial z}
\end{array}\right]\right)\right]^{\frac{1}{2}}
$$

Portanto,

$$
q=\sqrt{2\left(\frac{\partial u}{\partial r}\right)^{2}+2\left(\frac{\partial v}{\partial z}\right)^{2}+2\left(\frac{u}{r}\right)^{2}+\left(\frac{\partial u}{\partial z}+\frac{\partial v}{\partial r}\right)^{2}} .
$$


A viscosidade cinemática $\nu(q)$ é uma função conhecida da razão de cisalhamento representando a natureza "shear-thinning" do fluido. Um modelo muito usado para representar a viscosidade destes fluidos é o conhecido Cross Model dado por:

$$
\frac{\nu-\nu_{\infty}}{\nu_{0}-\nu_{\infty}}=\frac{1}{\left(1+(K q)^{m}\right)}
$$

sendo $m, \nu_{0}, \nu_{\infty}$ e $K$ constantes positivas dadas.

Com base no modelo Cross Model temos o seguinte gráfico do comportamento da viscosidade em função de $q$.

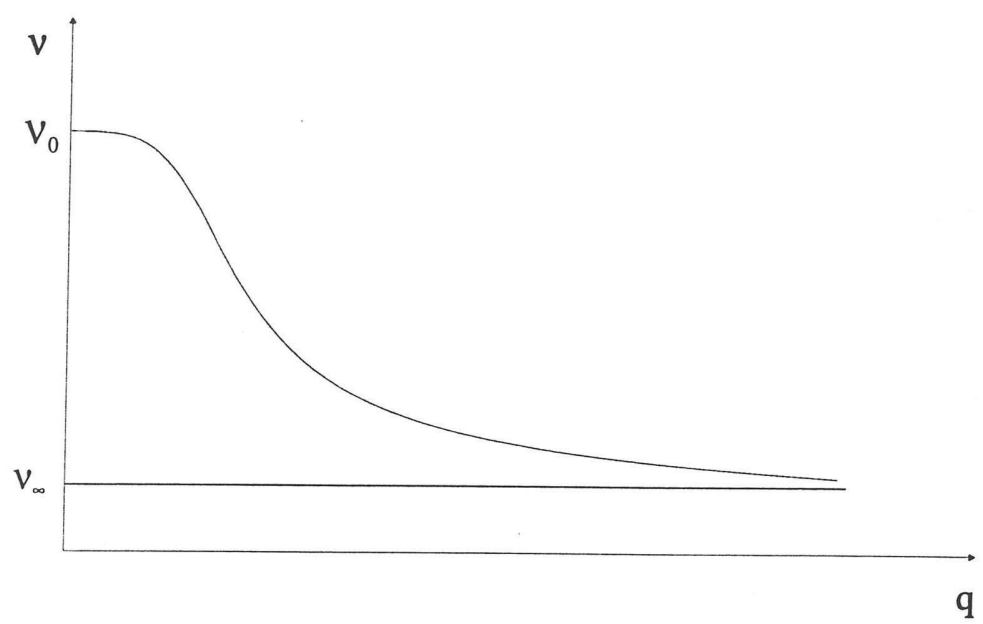

Fig. 1. Comportamento da viscosidade em função de $q$

\subsection{Condições de Contorno na Superfície Livre}

Neste trabalho nos ocuparemos de escoamentos transientes com superfícies livres de um fluido viscoso dentro de uma atmosfera passiva, na qual podemos tomar a pressão como sendo nula. Na ausência da tensão superficial as componentes de tensão normal e tangencial devem ser contínuas sobre tais superfícies e portanto:

$$
\mathbf{n} \cdot(\underline{\underline{\sigma}} \cdot \mathbf{n})=0, \quad \mathbf{m} \cdot(\underline{\underline{\sigma}} \cdot \mathbf{n})=0
$$

onde $\mathbf{n}=\left(n_{r}, n_{z}\right)$ é o vetor unitário normal exterior a superfície, $\mathbf{m}=\left(-n_{z}, n_{r}\right)$ é o vetor tangente e $\underline{\underline{\sigma}}$ é o tensor de tensão. 
Tem-se

$$
\underline{\underline{\sigma}} \cdot \mathbf{n}=\left[\begin{array}{c}
-p+2 \mu(q) \frac{\partial u}{\partial r} \mu(q)\left(\frac{\partial u}{\partial z}+\frac{\partial v}{\partial r}\right) \\
\mu(q)\left(\frac{\partial u}{\partial z}+\frac{\partial v}{\partial r}\right) p+2 \mu(q) \frac{\partial v}{\partial z}
\end{array}\right]\left[\begin{array}{c}
n_{r} \\
n_{z}
\end{array}\right]
$$

Calculando $\mathbf{n} \cdot(\underline{\underline{\sigma}} \cdot \mathbf{n})=0$ obtemos:

$$
\left(-p+2 \mu(q) \frac{\partial u}{\partial r}\right) n_{r}^{2}+2 \mu(q)\left(\frac{\partial u}{\partial z}+\frac{\partial v}{\partial r}\right) n_{z} n_{r}+\left(-p+2 \mu(q) \frac{\partial v}{\partial z}\right) n_{z}^{2}=0
$$

logo,

$$
-p\left(n_{r}^{2}+n_{z}^{2}\right)+2 \mu(q)\left[\frac{\partial u}{\partial r} n_{r}^{2}+\left(\frac{\partial u}{\partial z}+\frac{\partial v}{\partial r}\right) n_{z} n_{r}+\frac{\partial v}{\partial z} n_{z}^{2}\right]=0 .
$$

De forma análoga chegamos que $\mathbf{m} .(\underline{\underline{\sigma}} \cdot \mathbf{n})=0$ pode ser escrito como:

$$
\left[-2 n_{r} n_{z} \frac{\partial u}{\partial r}+\left(n_{r}^{2}-n_{z}^{2}\right)+\left(\frac{\partial u}{\partial z}+\frac{\partial v}{\partial r}\right)+2 n_{z} n_{r} \frac{\partial v}{\partial z}\right]=0 .
$$

Introduzindo a adimensionalização efetuada em (2.23) e (2.24), as condições de contorno na superfície livre podem ser escritas como:

$$
\begin{gathered}
\left.-p+\frac{2 \nu(q)}{R e}\left[\frac{\partial u}{\partial r} n_{r}^{2}+\left(\frac{\partial u}{\partial z}+\frac{\partial v}{\partial r}\right) n_{z} n_{r}+\frac{\partial v}{\partial z}\right) n_{z}^{2}\right]=0 \\
{\left[-2\left(\frac{\partial v}{\partial z}-\frac{\partial u}{\partial r}\right) n_{r} n_{z}+\left(\frac{\partial u}{\partial z}+\frac{\partial v}{\partial r}\right)\left(n_{r}^{2}-n_{z}^{2}\right)\right]=0}
\end{gathered}
$$

No capítulo 3 veremos como aproximar as condições de contorno para superfícies livrès considerando vários tipos de orientação da superfície.

Temos também as seguintes condições de fronteira para a velocidade:

$$
\begin{aligned}
& \mathbf{u}=0 \quad \text { na fronteira fixa ("condição no-slip") } \\
& u=U, \quad v=0 \quad \text { na fronteira "inflow" } \\
& \frac{\partial u}{\partial n}=0, \quad v=0 \quad \text { na fronteira "outflow" }
\end{aligned}
$$

onde $n$ denota a direção normal à fronteira. 


\section{Capítulo 3}

\section{Código GENSMAC}

Este capítulo apresenta um método numérico para resolver escoamentos axisimétricos de fluidos não-Newtonianos com superfícies livres. O método apresentado é uma extensão do método GENSMAC que é uma técnica de diferenças finitas aplicada sobre uma malha diferenciada e usa partículas marcadoras para a visualização do escoamento e localização da superfície livre do fluido.

\subsection{Características do Código}

O método GENSMAC consiste numa técnica de diferenças finitas aplicada sobre uma malha diferenciada, com o objetivo de investigar a dinâmica de um fluido incompressível. GENSMAC emprega variáveis primitivas de velocidade e pressão e usa partículas virtuais cujas coordenadas são armazenadas e atualizadas no tempo. O movimento destas partículas é efetuado de acordo com os valores mais recentes da velocidade. O método possui grande aplicação em modelagem de escoamentos de fluidos com superfícies livres.

Uma característica essencial usada em GENSMAC é que o ciclo de cálculo dividise em duas partes: uma para o cálculo do campo de velocidade e outra para o cálculo do campo de pressão. Desse modo, não existe nenhuma iteração envolvendo pressão e velocidade. Outro aspecto importante do método é o tratamento de escoamentos com superfícies livres em domínios arbitrários quando a condição "no-slip" é imposta em contornos rígidos curvos. O método GENSMAC como atualmente implementado tem as seguintes características:

(i) Foi escrito na linguagem FORTRAN 77 utilizando programação estruturada.

(ii) Tem capacidade para resolver escoamentos com superfícies livres em domínios bidimensionais arbitrários. O usuário precisa apenas fornecer os parâmetros de entrada das coordenadas e selecionar a função interpoladora mais apropriada para cada parte da curva.

(iii) Possui uma rotina que calcula o tamanho do passo no tempo $(\delta t)$, o que reduz o esforço computacional e o tempo de execução. 
(iv) Utiliza o método dos gradientes conjugados para resolver uma equação de Poisson para a obtenção do campo de velocidade atualizado.

(v) Possui uma boa aproximação para as condições de contorno na superfície livre do fluido.

(vi) Consegue simular escoamentos envolvendo muitas fronteiras do tipo "inflow" e "outflow".

\subsection{Algoritmo Computacional}

A idéia básica do método é resolver as equações de momento sobre uma malha diferenciada no tempo $t$ usando partículas marcadoras para representar o fluido. Uma vez encontrado o valor instantâneo de $\mathbf{u}(r, z, t)$, as posições das partículas são atualizadas resolvendo $d \mathbf{r} / d t=\mathbf{u}$ (sendo $\mathbf{u}=(u, v)^{T}$ e $\left.\mathbf{r}=(r, z)^{T}\right)$.

Supondo que em um dado tempo $t_{0}$ o campo de velocidade $\mathbf{u}\left(r, z, t_{0}\right)$ é conhecido e as condições de fronteira para velocidade e pressão são satisfeitas, o campo de velocidade atualizado $\mathbf{u}(r, z, t)$, onde $t=t_{0}+\delta t$ é determinado através dos seguintes passos:

(i) Usando $\mathbf{u}\left(r, z, t_{0}\right)$ calculamos $q\left(r, z, t_{0}\right)$ e $\nu\left(q\left(r, z, t_{0}\right)\right)$.

(ii) Seja $\tilde{p}\left(r, z, t_{0}\right)$ um campo de pressão arbitrário que satisfaça as condições de contorno na superfície livre em $t=t_{0}$. Este campo de pressão é calculado de acordo com a condição de contorno na superficie livre e é obtido conforme as equações descritas na Seç̧ão (4.5).

(iii) Calcula-se um campo de velocidade intermediário $\widetilde{\mathbf{u}}(r, z, t)$ de $(2.16)$, ou seja,

$$
\frac{\partial \widetilde{\mathbf{u}}}{\partial t}=-\nabla \widetilde{p}+\mathbf{N}(\mathbf{u})
$$

onde $\mathbf{N}(\mathbf{u})$ é definida por (2.17) e (2.18). Este campo de velocidade deve satisfazer a condição inicial $\tilde{\mathbf{u}}\left(r, z, t_{0}\right)=\mathbf{u}\left(r, z, t_{0}\right)$. Para obter-se $\tilde{\mathbf{u}}(r, z, t)$, equação (3.1) é resolvida numéricamente utilizando-se o método de Euler.

Subtraindo (3.1) de (2.16) obtemos

$$
\frac{\partial}{\partial t}(\mathbf{u}-\widetilde{\mathbf{u}})=-\nabla(p-\widetilde{p}) .
$$

Tomando o rotacional em ambos os lados vem

$$
\nabla \times\left[\frac{\partial}{\partial t}(\mathbf{u}-\widetilde{\mathbf{u}})\right]=\nabla \times(-\nabla(p-\tilde{p})),
$$


$\log \mathrm{O}$

$$
\nabla \times\left[\frac{\partial}{\partial t}(\mathbf{u}-\widetilde{\mathbf{u}})\right]=0 .
$$

Mudando a ordem dos operadores obtém-se:

$$
\frac{\partial}{\partial t}[\nabla \times(\mathbf{u}-\widetilde{\mathbf{u}})]=0
$$

implicando em $\nabla \times(\mathbf{u}-\widetilde{\mathbf{u}})=f(x)$ para alguma $f(x), t \in\left[t_{0}, t_{0}+\delta t\right]$. Como $\widetilde{\mathbf{u}}=\mathbf{u}$ em $t=t_{0}$ temos que $\nabla \times \mathbf{u}=\nabla \times \widetilde{\mathbf{u}}$ em $t=t_{0}$, implicando em $f(x)=0$. Logo, $\nabla \times(\mathbf{u}-\widetilde{\mathbf{u}})=0, \quad \forall t \in\left[t_{0}, t_{0}+\delta t\right]$.

Isso mostra que as "vorticidades" associadas com $\widetilde{\mathbf{u}}(r, z, t)$ e $\mathbf{u}(r, z, t)$ são iguais. Porém, $\widetilde{\mathbf{u}}(r, z, t)$ não satisfaz $\nabla \cdot \widetilde{\mathbf{u}}=0$. Desta forma existe uma função escalar $\psi(r, z, t)$ tal que

$$
\mathbf{u}(r, z, t)-\widetilde{\mathbf{u}}(r, z, t)=-\nabla \psi(r, z, t) .
$$

Aplicando o divergente à equação acima e impondo continuidade obtemos

$$
\nabla^{2} \psi(r, z, t)=\nabla \cdot \widetilde{\mathbf{u}}(r, z, t) .
$$

(iv) Resolver a equação de Poisson.

$$
\nabla^{2} \psi(r, z, t)=\nabla \cdot \widetilde{\mathbf{u}}(r, z, t)
$$

As condições de fronteira para $\psi$ são:

$$
\frac{\partial \psi}{\partial n}=0 \quad \text { na fronteira rígida, e } \psi=0 \text { na superfície livre, }
$$

onde $n$ é a direção normal ao contorno rígido.

A primeira condição ocorre devido a imposição das condições de contorno "no-slip" ou "free-slip", sendo que nesses casos, a velocidade normal ao contorno deve ser nula. De fato, da equação (3.3) vemos que:

$$
-\nabla \psi(r, z, t)=\mathbf{u}(r, z, t)-\widetilde{\mathbf{u}}(r, z, t)
$$

como $\widetilde{\mathbf{u}}(r, z, t)$ satisfaz as mesmas condições de contorno impostas para $\mathbf{u}(r, z, t)$ e tendo em vista que consideraremos domínios retangulares (pois qualquer domínio curvo pode ser decomposto em um outro que coincida com os seguimentos da malha (fronteira virtual) Tome and McKee [7]) obtemos:

$\frac{\partial \psi}{\partial n}=0, \quad$ onde $n=r$ ou $n=z$. 
A segunda condição se deve ao fato de que a função $\widetilde{p}$ (ver eq. (3.6)) satisfaz perfeitamente a condição de pressão sobre a superfície livre, como $p=\tilde{p}+\frac{\psi}{\delta t}$ (como veremos a seguir) isto implica em $\psi=0$.

(v) Obter o campo de velocidade atualizado $\mathbf{u}(r, z, t)$.

De (3.3) tem-se

$$
\mathbf{u}(r, z, t)=\widetilde{\mathbf{u}}(r, z, t)-\nabla \psi(r, z, t)
$$

(vi) Calcular a pressão

Calculada $\mathbf{u}(r, z, t)$, a pressão segue das equações (3.2) e (3.3). De fato, substituindo $\mathbf{u}(r, z, t)-\widetilde{\mathbf{u}}(r, z, t)$ de (3.3) em (3.2), vem:

$$
\frac{\partial}{\partial t}(-\nabla \psi(r, z, t))=-\nabla(p-\tilde{p})
$$

e mudando a ordem dos operadores obtem-se:

$$
-\nabla\left(\frac{\partial \psi(r, z, t)}{\partial t}\right)=-\nabla(p-\widetilde{p})
$$

Logo,

$$
\frac{\partial \psi(r, z, t)}{\partial t}=p-\widetilde{p}
$$

que pode ser aproximada por

$$
\frac{\psi(r, z, t)-\psi\left(r, z, t_{0}\right)}{\delta t}=p-\tilde{p}
$$

Mas, para $t=t_{0}$ tem-se que $\mathbf{u}\left(r, z, t_{0}\right)=\widetilde{\mathbf{u}}\left(r, z, t_{0}\right)$, e por (3.3) vemos que

$$
\nabla \psi\left(r, z, t_{0}\right)=0 \quad \longrightarrow \quad \psi\left(r, z, t_{0}\right) \quad \text { é constante. }
$$

Tomando $\psi\left(r, z, t_{0}\right)=0$ em (3.5), $p$ é avaliada numericamente como

$$
p=\widetilde{p}+\frac{\psi(r, z, t)}{\delta t} .
$$

onde $\delta t$ é o time-step. Desta forma, a equação de momento é resolvida explícitamente e um sistema linear simétrico e esparso (a equação de Poisson discreta) para a obtenção da velocidade potencial $\nabla \psi$.

(vii) Atualização das posições das partículas marcadoras

O último passo envolve o movimento das partículas marcadoras para sua nova posição. Essas partículas são utilizadas para representar o fluido. Elas são criadas nos "inlets" e injetadas no domínio, fornecendo assim a visualização instantânea do escoamento e obtenção da orientação da superfície livre. No final de cada ciclo, as 
posições destas partículas são atualizadas de acordo com os valores atualizados da velocidade. O movimento destas partículas é feito resolvendo-se as equações

$$
\frac{d r}{d t}=u \quad e \quad \frac{d z}{d t}=v
$$

pelo método Euler. Isto fornece as novas coordenadas da partícula determinando se a partícula move-se para dentro de uma nova célula ou deixa a região de domínio do fluido através de um "outflow".

\subsection{Cálculo da Shear Rate na Fronteira Rígida}

Quando calculamos a velocidade $\tilde{\mathbf{u}}$ de (3.1), os valores da shear rate na fronteira rígida são necessários. Estes valores podem ser obtidos da seguinte forma:

Consideremos um contorno rígido horizontal, $z=z_{c}$. Então se a condição "no-slip" for imposta neste contorno, tem-se:

$$
u\left(r, z_{c}\right)=0 \quad \text { e } \quad v\left(r, z_{c}\right)=0, \quad \forall r,
$$

que produz

$$
\frac{\partial u}{\partial r}=0 \quad \text { e } \quad \frac{\partial v}{\partial r}=0 \quad \text { para } z=z_{c} .
$$

Introduzindo estas condições à equação (2.20), obtemos a "shear rate" em um contorno horizontal "no-slip" como:

$$
q\left(r, z_{c}\right)=\left[2\left(\frac{\partial v}{\partial z}\right)^{2}+\left(\frac{\partial u}{\partial z}\right)^{2}+2\left(\frac{u}{r}\right)^{2}\right]^{1 / 2} .
$$

Por outro lado se a condição "free-slip" for imposta em $z=z_{c}$, temos

$$
v\left(r, z_{c}\right)=0 \quad \text { e } \quad \frac{\partial u}{\partial z}=0 \quad \text { para } z=z_{c}, \quad \forall r
$$

donde obtêm-se

$$
\frac{\partial v}{\partial r}=0 \quad \text { para } z=z_{c} .
$$

Novamente, introduzindo estas equações em (2.20), obtemos

$$
q\left(r, z_{c}\right)=\left[2\left(\left(\frac{\partial u}{\partial r}\right)^{2}+\left(\frac{\partial v}{\partial z}\right)^{2}+\left(\frac{u}{r}\right)^{2}\right)\right]^{1 / 2}
$$

Agora, considerando um contorno rígido vertical e impondo a condição "no-slip" em $r=r_{c}$, vem:

$$
u\left(r_{c}, z\right)=0 \quad \text { e } \quad v\left(r_{c}, z\right)=0, \forall z,
$$

fornecendo

$$
\frac{\partial u}{\partial z}=0 \quad \text { e } \quad \frac{\partial v}{\partial z}=0 \quad \text { para } \quad r=r_{c}
$$


Introduzindo estas equações em (2.20), temos:

$$
q\left(r_{c}, z\right)=\left[2\left(\left(\frac{\partial u}{\partial r}\right)^{2}+\left(\frac{u}{r}\right)^{2}\right)+\left(\frac{\partial v}{\partial r}\right)^{2}\right]^{1 / 2}
$$

Por outro lado se a condição "free-slip" for imposta em $r=r_{c}$, tem-se

$$
u\left(r_{c}, z\right)=0 \quad \text { e } \quad \frac{\partial v}{\partial r}=0 \quad \text { sobre } \quad r=r_{c}, \forall z
$$

que produz

$$
\frac{\partial u}{\partial z}=0 \quad \text { em } \quad r=r_{c} .
$$

Mais uma vez, introduzindo estas equações em (2.20), obtemos:

$$
q\left(r_{c}, z\right)=\left[2\left(\left(\frac{\partial u}{\partial r}\right)^{2}+\left(\frac{\partial v}{\partial z}\right)^{2}+\left(\frac{u}{r}\right)^{2}\right)\right]^{1 / 2}
$$

No capítulo a seguir apresentaremos o método de diferenças finitas para obter uma solução aproximada do procedimento apresentado nesse capítulo. 


\section{Capítulo 4}

\section{Aproximação por Diferenças Finitas}

Este capítulo apresenta as equações desenvolvidas no capítulo 3 aproximadas por diferenças finitas. Também mostra o estudo sobre as condições de contorno nas superfícies livres, uma rotina time-step e detalhes sobre a solução da equação de Poisson.

A implementação do método apresentado no capítulo anterior é feita usando a técnica de diferenças finitas da seguinte forma:

Uma malha diferenciada com células de dimensões $\delta r$ por $\delta z$ é empregada. No centro das células são posicionadas as variáveis de pressão $\widetilde{p}_{i, j}$, velocidade potencial $\psi_{i, j}$, divergente $\widetilde{D}_{i, j}$ e a shear rate discreta $q_{i, j}$, (como mostra a figura 1 ); enquanto as velocidades $u$ e $v$ são posicionadas nos lados da célula nas posições $i+1 / 2$ e $j+1 / 2$, respectivamente.

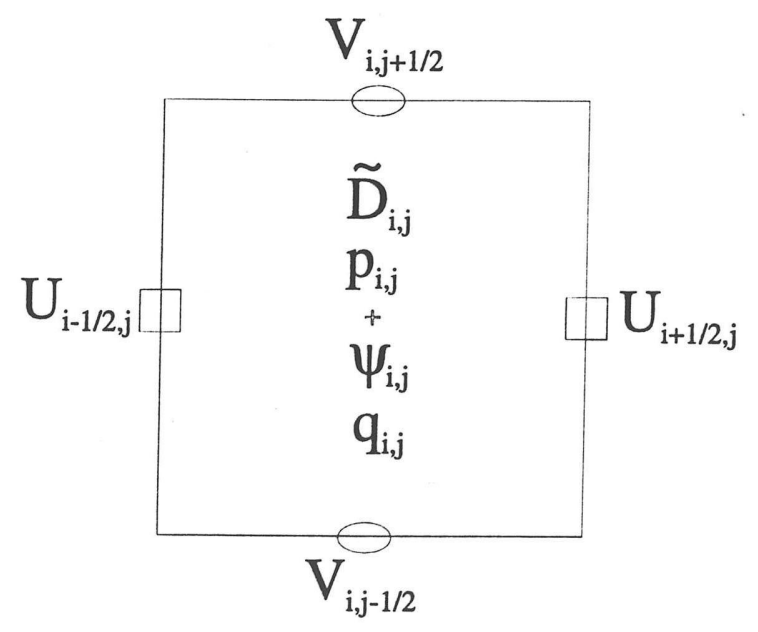

Fig. 2. Célula Computacional.

A discretização da equação de momento (3.1) para obtenção de $\widetilde{u}(r, z, t)$ é aplicada e aproximada nos nós $u$ e $v$, respectivamente. 


\subsection{Cálculo de $u_{i+\frac{1}{2}, j}^{n+1}$}

Por exemplo, consideremos a equação (3.1) para o cálculo de $\widetilde{\mathbf{u}}(r, z, t)$. Para obtermos uma aproximação para $\widetilde{\mathbf{u}}(r, z, t)$ procederemos como segue:

As derivadas em função do tempo são aproximadas usando diferenças avançadas enquanto que os termos que não contém $\nu$-derivadas são aproximados usando diferenças centrais, ou seja,

$$
\begin{gathered}
{\left[\frac{\partial u}{\partial t}\right]_{i+1 / 2, j}=\left(\frac{\left.u_{i+1 / 2, j}^{n+1}-u_{i+1 / 2, j}^{n}\right),}{\delta t}\right)} \\
{\left[\frac{\partial p}{\partial r}\right]_{i+1 / 2, j}=\left(\frac{\widetilde{p}_{i+1, j}-\widetilde{p}_{i, j}}{\delta r}\right),} \\
{\left[\frac{1}{r} \frac{\partial\left(r u^{2}\right)}{\partial r}\right]_{i+1 / 2, j}=\frac{1}{r_{i+1 / 2}} \frac{r_{i+1} u_{i+1, j}^{2}-r_{i} u_{i, j}^{2}}{\delta r}}
\end{gathered}
$$

onde $\left(u_{i, j}\right)^{2}$ é aproximado adotando a forma ZIP:

$$
\left(u_{i, j}\right)^{2}=u_{i+1 / 2, j} u_{i-1 / 2, j}
$$

Logo,

$$
\left[\frac{1}{r} \frac{\partial\left(r u^{2}\right)}{\partial r}\right]_{i+1 / 2, j}=\frac{1}{r_{i+1 / 2}} \frac{r_{i+1}\left(u_{i+3 / 2, j} u_{i+1 / 2, j}\right)-r_{i}\left(u_{i+1 / 2, j} u_{i-1 / 2, j}\right)}{\delta r} .
$$

Para os termos de fluxo $\frac{\partial(u v)}{\partial z}$, usamos diferenças centradas após ter sido feita uma média aritmética para obter $(u v)$ nos cantos $(i+1 / 2, j+1 / 2)$ e $(i+1 / 2, j-1 / 2)$ da célula.

$$
\left[\frac{\partial(u v)}{\partial z}\right]_{i+1 / 2, j}=\frac{(u v)_{i+1 / 2, j+1 / 2}-(u v)_{i+1 / 2, j-1 / 2}}{\delta z}
$$

sendo

e

$$
(u v)_{i+1 / 2, j+1 / 2}=\frac{\left(u_{i+1 / 2, j}+u_{i+1 / 2, j+1}\right)}{2} \frac{\left(v_{i, j+1 / 2}+v_{i+1, j+1 / 2}\right)}{2}
$$

Assim,

$$
(u v)_{i+1 / 2, j-1 / 2}=\frac{\left(u_{i+1 / 2, j}+u_{i+1 / 2, j-1}\right)}{2} \frac{\left(v_{i, j-1 / 2}+v_{i+1, j-1 / 2}\right)}{2}
$$

$$
\begin{aligned}
{\left[\frac{\partial(u v)}{\partial z}\right]_{i+1 / 2, j}=} & \frac{1}{4 \delta z}\left[\left(u_{i+1 / 2, j}+u_{i+1 / 2, j+1}\right)\left(v_{i, j+1 / 2}+v_{i+1, j+1 / 2}\right)\right. \\
& \left.-\left(u_{i+1 / 2, j-1}+u_{i+1 / 2, j}\right)\left(v_{i, j-1 / 2}+v_{i+1, j-1 / 2}\right)\right] .
\end{aligned}
$$

Para aproximar o termo $\frac{\nu(q)}{R e} \frac{\partial}{\partial z}\left(\frac{\partial u}{\partial z}-\frac{\partial v}{\partial r}\right)$ da equação (3.1) (conforme eq. (2.17)) são obtidas as seguintes aproximações para as derivadas: 
e

$$
\begin{gathered}
\frac{\partial}{\partial z}\left[\frac{\partial u}{\partial z}\right]_{i+1 / 2, j}=\left[\frac{\partial^{2} u}{\partial z^{2}}\right]_{i+1 / 2, j} \\
{\left[\frac{\partial^{2} u}{\partial z^{2}}\right]_{i+1 / 2, j}=\frac{u_{i+1 / 2, j+1}-2 u_{i+1 / 2, j}+u_{i+1 / 2, j-1}}{(\delta z)^{2}}}
\end{gathered}
$$

$\operatorname{logo}$

$$
\begin{gathered}
\frac{\partial}{\partial z}\left[\frac{\partial v}{\partial r}\right]_{i+1 / 2, j}=\frac{\partial}{\partial z}\left[\frac{v_{i+1, j}-v_{i, j}}{\delta r}\right]_{i+1 / 2, j} \\
\frac{\partial}{\partial z}\left[\frac{v_{i+1, j}-v_{i, j}}{\delta r}\right]_{i+1 / 2, j}=\frac{\left(\frac{\partial v}{\partial z}\right)_{i+1, j}-\left(\frac{\partial v}{\partial z}\right)_{i, j}}{\delta r}
\end{gathered}
$$

$$
\frac{\partial}{\partial z}\left[\frac{\partial v}{\partial r}\right]_{i+1 / 2, j}=\frac{v_{i+1, j+1 / 2}-v_{i+1, j-1 / 2}+v_{i, j-1 / 2}-v_{i, j+1 / 2}}{\delta z \delta r} .
$$

Para o termo $\frac{1}{R e}\left[2 \frac{\partial u}{\partial r} \frac{\partial \nu}{\partial r}+\left(\frac{\partial u}{\partial z}+\frac{\partial v}{\partial r}\right) \frac{\partial \nu}{\partial z}\right]$, obtém-se as seguintes aproximações para as derivadas de $u$ e $v$.

$$
\begin{aligned}
& {\left[\frac{\partial u}{\partial r}\right]_{i+1 / 2, j}=\frac{u_{i+3 / 2, j}-u_{i-1 / 2, j}}{2 \delta r}} \\
& {\left[\frac{\partial u}{\partial z}\right]_{i+1 / 2, j}=\frac{u_{i+1 / 2, j+1}-u_{i+1 / 2, j-1}}{2 \delta z}} \\
& {\left[\frac{\partial v}{\partial r}\right]_{i+1 / 2, j}=\frac{v_{i+1, j}-v_{i, j}}{\delta r}}
\end{aligned}
$$

onde $v_{i+1, j}=\frac{1}{2}\left(v_{i+1, j+1 / 2}+v_{i+1, j-1 / 2}\right)$ e $v_{i, j}=\frac{1}{2}\left(v_{i, j+1 / 2}+v_{i, j-1 / 2}\right)$.

Desta forma:

$$
\left[\frac{\partial v}{\partial r}\right]_{i+1 / 2, j}=\frac{v_{i+1, j+1 / 2}+v_{i+1, j-1 / 2}-v_{i, j+1 / 2}-v_{i, j-1 / 2}}{2 \delta r} .
$$

\subsubsection{Discretização da Shear Rate}

Aplicando a equação da "shear rate" no centro da célula temos:

$$
q_{i, j}=\sqrt{2\left(\frac{\partial u}{\partial r}\right)_{i, j}^{2}+2\left(\frac{\partial v}{\partial z}\right)_{i, j}^{2}+2\left(\frac{u}{r}\right)_{i, j}^{2}+\left(\frac{\partial u}{\partial z}+\frac{\partial v}{\partial r}\right)_{i, j}^{2}}
$$

Os termos $\frac{\partial u}{\partial r}$ e $\frac{\partial v}{\partial z}$ são aproximados por diferenças centradas e para o termo $\left(\frac{u}{r}\right)^{2}$ foi utilizada a forma ZIP. Logo,

$$
\left[\frac{\partial u}{\partial r}\right]_{i, j}=\frac{u_{i+1 / 2, j}-u_{i-1 / 2, j}}{\delta r}
$$




$$
\begin{gathered}
{\left[\frac{\partial v}{\partial z}\right]_{i, j}=\frac{v_{i, j+1 / 2}-v_{i, j-1 / 2}}{\delta z}} \\
{\left[\frac{u^{2}}{r^{2}}\right]_{i, j}=\frac{u_{i+1 / 2, j} u_{i-1 / 2, j}}{r_{i}^{2}} .}
\end{gathered}
$$

Para aproximar os termos $\frac{\partial u}{\partial z}$ e $\frac{\partial v}{\partial r}$ primeiro é feito o cálculo de uma média, em seguida aplicamos diferenças centradas, ou seja,

$$
\left[\frac{\partial u}{\partial z}\right]_{i, j}=\frac{u_{i, j+1 / 2}-u_{i, j-1 / 2}}{\delta z}
$$

onde

$$
\begin{aligned}
& u_{i, j+1 / 2}=\frac{u_{i+1 / 2, j+1}+u_{i-1 / 2, j+1}+u_{i+1 / 2, j},+u_{i-1 / 2, j}}{4} \\
& u_{i, j-1 / 2}=\frac{u_{i+1 / 2, j}+u_{i-1 / 2, j}+u_{i+1 / 2, j-1}+u_{i-1 / 2, j-1}}{4}
\end{aligned}
$$

e

$$
\left[\frac{\partial v}{\partial r}\right]_{i, j}=\frac{v_{i+1 / 2, j}-v_{i-1 / 2, j}}{\delta r}
$$

onde

$$
\begin{aligned}
& v_{i+1 / 2, j}=\frac{v_{i+1, j+1 / 2}+v_{i, j+1 / 2}+v_{i+1, j-1 / 2}+v_{i, j-1 / 2}}{4}, \\
& v_{i-1 / 2, j}=\frac{v_{i, j+1 / 2}+v_{i, j-1 / 2}+v_{i-1, j+1 / 2}+v_{i-1, j-1 / 2}}{4} .
\end{aligned}
$$

Logo a shear rate $q_{i, j}$ é aproximada por:

$$
\begin{aligned}
q_{i, j}= & {\left[2 \frac{\left(u_{i+1 / 2, j}-u_{i-1 / 2, j}\right)^{2}}{\delta r^{2}}+2 \frac{\left(v_{i, j+1 / 2}-v_{i, j-1 / 2}\right)^{2}}{\delta z^{2}}\right.} \\
& +2 \frac{\left(u_{i+1 / 2, j} u_{i-1 / 2, j}\right)}{r_{i}^{2}}+\left(\frac{u_{i+1 / 2, j+1}+u_{i-1 / 2, j+1}-u_{i+1 / 2, j-1}-u_{i-1 / 2, j-1}}{4 \delta z}\right. \\
& \left.\left.+\frac{v_{i+1, j+1 / 2}+v_{i+1, j-1 / 2}-v_{i-1, j+1 / 2}-v_{i-1, j-1 / 2}}{4 \delta r}\right)^{2}\right]^{\frac{1}{2}} \cdot
\end{aligned}
$$

Uma vez obtido $q_{i, j}$, calcula-se agora as $\nu$-derivadas da equação (3.1) (conforme eq. (2.17)), da seguinte forma: Consideremos por exemplo, uma célula full com vizinhanças diferentes de célula empty. O termo $\frac{\partial \nu}{\partial r}$ é aproximado por diferenças centradas e para o termo $\frac{\partial \nu}{\partial z}$ primeiro faz-se uma média aritmética para obter a "shear rate" nos cantos da célula e aplica-se diferenças centradas, como segue:

$$
\left[\frac{\partial \nu}{\partial r}\right]_{i+1 / 2, j}=\frac{\left(\nu\left(q_{i+1, j}\right)-\nu\left(q_{i, j}\right)\right)}{\delta r}
$$




$$
\left[\frac{\partial \nu}{\partial z}\right]_{i+1 / 2, j}=\frac{\left(\nu\left(q_{i+1 / 2, j+1 / 2}\right)-\nu\left(q_{i+1 / 2, j-1 / 2}\right)\right)}{\delta z}
$$

onde

$$
\begin{aligned}
q_{i+1 / 2, j+1 / 2} & =\frac{q_{i+1, j}+q_{i, j}+q_{i+1, j+1}+q_{i, j+1}}{4}, \\
q_{i+1 / 2, j-1 / 2} & =\frac{q_{i+1, j}+q_{i, j}+q_{i+1, j-1}+q_{i, j-1}}{4} .
\end{aligned}
$$

\subsubsection{Cálculo da Viscosidade}

Empregando o modelo "Cross Model" o valor da viscosidade $\nu(q)$ no nó $u$ é calculado por:

$$
\nu\left(q_{i+1 / 2, j}\right)=\nu_{\infty}+\frac{\nu_{0}-\nu_{\infty}}{\left(1+\left(K q_{i+1 / 2, j}\right)^{m}\right)},
$$

onde $q_{i+1 / 2, j}=\frac{q_{i+1, j}+q_{i, j}}{2}$.

Introduzindo as aproximações desenvolvidas neste seção para o cálculo de $\widetilde{u}$ (conforme as equações (3.1) e (2.17)), o valor de $\tilde{u}_{i+1 / 2, j}^{n+1}$ é calculado por:

$$
\begin{aligned}
\tilde{u}_{i+1 / 2, j}^{n+1}= & \tilde{u}_{i+1 / 2, j}^{n}+\delta t\left[\frac{u_{i+1 / 2, j}}{r_{i+1 / 2} \delta r}\left(r_{i} u_{i-1 / 2, j}-r_{i+1} u_{i+3 / 2, j}\right)\right. \\
& +\frac{1}{4 \delta z}\left(\left(u_{i+1 / 2, j-1}+u_{i+1 / 2, j}\right)\left(v_{i, j-1 / 2}+v_{i+1, j-1 / 2}\right)\right. \\
& \left.-\left(u_{i+1 / 2, j}+u_{i+1 / 2, j+1}\right)\left(v_{i, j+1 / 2}+v_{i+1, j+1 / 2}\right)\right) \\
& +\frac{p_{i, j}-p_{i+1, j}}{\delta r}+\frac{\nu\left(q_{i+1 / 2, j}\right)}{R e}\left(\frac{u_{i+1 / 2, j+1}-2 u_{i+1 / 2, j}+u_{i+1 / 2, j-1}}{(\delta z)^{2}}\right. \\
& \left.-\frac{v_{i+1, j+1 / 2}-v_{i+1, j-1 / 2}+v_{i, j-1 / 2}-v_{i, j+1 / 2}}{\delta z \delta r}\right)+\frac{1}{F r^{2}} g r \\
& +\frac{1}{R e}\left(\frac{u_{i+3 / 2, j}-u_{i-1 / 2, j}}{\delta r} \frac{\partial \nu\left(q_{i+1 / 2, j}\right)}{\delta r}+\left(\frac{u_{i+1 / 2, j+1}-u_{i+1 / 2, j-1}}{2 \delta z}\right.\right. \\
& \left.\left.\left.+\frac{v_{i+1, j+1 / 2}+v_{i+1, j-1 / 2}-v_{i, j+1 / 2}-v_{i, j-1 / 2}}{2 \delta r}\right) \frac{\partial \nu\left(q_{i+1 / 2, j}\right)}{\delta z}\right)\right] .
\end{aligned}
$$




\subsection{Cálculo de $v_{i, j+\frac{1}{2}}^{n+1}$}

De maneira análoga, obtém-se a seguinte aproximação para o cálculo de $\tilde{v}_{i, j+1 / 2}^{n+1}$

$$
\begin{aligned}
\tilde{v}_{i, j+1 / 2}^{n+1}= & \widetilde{v}_{i, j+1 / 2}^{n}+\delta t\left[\frac { 1 } { 4 r _ { i } } \left(r_{i-1 / 2}\left(u_{i-1 / 2, j+1}+u_{i-1 / 2, j}\right)\left(v_{i-1, j+1 / 2}+v_{i, j+1 / 2}\right)\right.\right. \\
& \left.-r_{i+1 / 2}\left(u_{i+1 / 2, j}+u_{i+1 / 2, j+1}\right)\left(v_{i, j+1 / 2}+v_{i+1, j+1 / 2}\right)\right) \\
& +\frac{v_{i, j+1 / 2}}{\delta z}\left(v_{i, j-1 / 2}-v_{i, j+3 / 2}\right)+\frac{p_{i, j}-p_{i, j+1}}{\delta z} \\
& -\frac{1}{R e} \frac{\nu\left(q_{i, j+1 / 2}\right)}{r_{i} \delta r}\left(r_{i+1 / 2}\left(\frac{u_{i+1 / 2, j+1}-u_{i+1 / 2, j}}{\delta z}-\frac{v_{i+1, j+1 / 2}-v_{i, j+1 / 2}}{\delta r}\right)\right. \\
& \left.-r_{i-1 / 2}\left(\frac{u_{i-1 / 2, j+1}-u_{i-1 / 2, j}}{\delta z}-\frac{v_{i, j+1 / 2}-v_{i-1, j+1 / 2}}{\delta r}\right)\right) \\
& +\frac{1}{R e}\left(\left(\frac{u_{i+1 / 2, j+1}+u_{i-1 / 2, j+1}-u_{i+1 / 2, j}-u_{i-1 / 2, j}}{2 \delta z}\right)\right. \\
& \left.\left.\left.+\frac{v_{i+1, j+1 / 2}-v_{i-1, j+1 / 2}}{\delta r}\right) \frac{\partial \nu\left(q_{i, j+1 / 2}\right)}{\delta r}+\frac{v_{i, j+3 / 2}-v_{i, j-1 / 2}}{\delta z} \frac{\partial \nu\left(q_{i, j+1 / 2}\right)}{\delta z}\right)\right]
\end{aligned}
$$

onde $(\partial \nu / \partial r)_{i, j+1 / 2},(\partial \nu / \partial z)_{i, j+1 / 2}, \nu\left(q_{i, j+1 / 2}\right)$ e $q_{i, j+1 / 2}$ são obtidos de maneira semelhante aos cálculos efetuados para a obtenção de $(\partial \nu / \partial r)_{i+1 / 2, j},(\partial \nu / \partial z)_{i+1 / 2, j}, \nu\left(q_{i+1 / 2, j}\right)$ e $q_{i+1 / 2, j}$, com aproximações adequadas de acordo com suas vizinhanças.

\subsection{Aproximação da Equação de Poisson}

Tomando a equação $\nabla^{2} \psi(r, z, t)=\widetilde{D}$ e aplicando-a sobre a malha diferenciada vem:

$$
\left(\frac{1}{r} \frac{\partial}{\partial r}\left(r \frac{\partial \psi}{\partial r}\right)+\frac{\partial^{2} \psi}{\partial z^{2}}\right)_{i, j}=\widetilde{D}_{i, j}
$$

ou seja

$$
\left(\frac{\partial}{\partial r}\left(r \frac{\partial \psi}{\partial r}\right)\right)_{i, j}+r_{i}\left(\frac{\partial^{2} \psi}{\partial z^{2}}\right)_{i, j}=r_{i} \widetilde{D}_{i, j}
$$

Aproximando o termo $\frac{\partial}{\partial r}\left(r \frac{\partial \psi}{\partial r}\right)_{i, j}$ por diferenças centrais temos:

$$
\frac{\partial}{\partial r}\left(r \frac{\partial \psi}{\partial r}\right)_{i, j}=\frac{r_{i+1 / 2}\left(\frac{\partial \psi}{\partial r}\right)_{i+1 / 2, j}-r_{i-1 / 2}\left(\frac{\partial \psi}{\partial r}\right)_{i-1 / 2, j}}{\delta r}
$$




$$
\begin{aligned}
= & \frac{1}{\delta r}\left[r_{i+1 / 2}\left(\frac{\psi_{i+1, j}-\psi_{i, j}}{\delta r}\right)-r_{i-1 / 2}\left(\frac{\psi_{i, j}-\psi_{i-1, j}}{\delta r}\right)\right] \\
= & \frac{1}{(\delta r)^{2}}\left[r_{i+1 / 2} \psi_{i+1, j}-\psi_{i, j}\left(r_{i+1 / 2}+r_{i-1 / 2}\right)+r_{i-1 / 2, j} \psi_{i-1, j}\right]
\end{aligned}
$$

Portanto

$$
\frac{\partial}{\partial r}\left(r \frac{\partial \psi}{\partial r}\right)_{i, j}=\frac{1}{(\delta r)^{2}}\left[r_{i+1 / 2} \psi_{i+1, j}-2 r_{i} \psi_{i, j}+r_{i-1 / 2} \psi_{i-1, j}\right]
$$

De maneira análoga, aplicando diferenças centrais ao termo $\left.\frac{\partial^{2} \psi}{\partial z^{2}}\right|_{i, j}$ obtêm-se

$$
\left.\frac{\partial^{2} \psi}{\partial z^{2}}\right|_{(i, j)}=\frac{\psi_{i, j+1}-2 \psi_{i, j}+\psi_{i, j-1}}{\delta z^{2}}
$$

Fazendo $\delta r=\delta z=h$ e substituindo (4.6) e (4.7) na equação de Poisson (4.5) temos:

$$
-r_{i} \psi_{i, j-1}-r_{i-1 / 2} \psi_{i-1, j}+4 r_{i} \psi_{i, j}-r_{i+1 / 2} \psi_{i+1, j}-r_{i} \psi_{i, j+1}=r_{i} \widetilde{D}_{i, j}
$$

onde $\widetilde{D}_{i, j}$ é definido por:

$$
\widetilde{D}_{i, j}=\frac{1}{r_{i}}\left(\frac{r_{i+1 / 2} \widetilde{u}_{i+1 / 2, j}-r_{i-1 / 2} \widetilde{u}_{i-1 / 2, j}}{\delta r}\right)+\frac{\widetilde{v}_{i, j+1 / 2}-\widetilde{v}_{i, j-1 / 2}}{\delta z} .
$$

Pode-se mostrar que a equação de Poisson discreta (4.8) tem um erro de truncamento $O\left(h^{2}\right)$.

\subsection{Definição das Células}

Como o fluido está continuamente em movimento, um procedimento para identificar a região contendo o fluido e a superfície livre é empregado. Para isto, as células da malha são rotuladas da seguinte maneira (ver figura 3).

1) Células Boundary (B) - São células que definem a localização do contorno rígido de maneira que as condições de contorno possam ser impostas.

2) Células Empty (E) - São células que não contém fluido.

3) Células Full (F) - São células que contém fluido e não possuem nenhuma adjacência com uma célula empty.

4) Células Surface (S) - São células que contém fluido e têm pelo menos uma adjacência com uma célula empty. Essas células definem a posição e orientação da superfície livre. Nestas células as condições de fronteira na superfície livre são impostas. 
5) Células Inflow (I) - São células que simulam a entrada de fluido na região de domínio do fluido. Estas células definem um contorno do tipo "inflow".

6) Células Outflow (O) - São células que simulam a saída de fluido da região de domínio do fluido. Essas células definem um contorno do tipo "outflow".

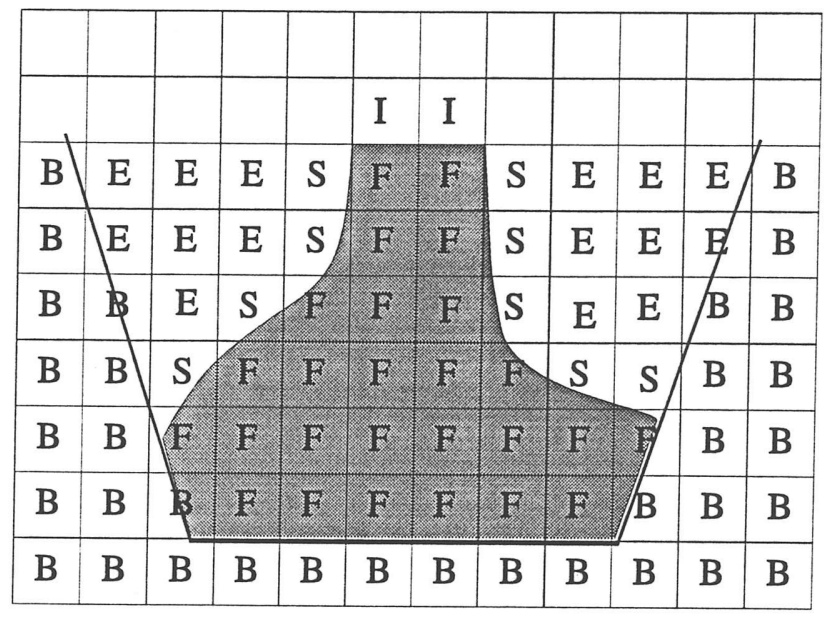

Fig. 3. Tipos de célula existentes na malha.

\subsection{Aproximação das Condições de Contorno na Su- perfície Livre}

A condição de contorno na superfície livre representada pelas equações (2.25) e (2.26) (ver Capítulo 2), serão aproximadas por diferenças finitas locais assumindo três tipos de orientação para a superfície livre: horizontal, vertical e superfície inclinada à $45^{\circ}$. Nesse ponto, gostaríamos de reapresentar as equações de contorno na superfície livre obtidas no Capítulo 2

$$
\begin{gathered}
\left.-p+\frac{2 \nu(q)}{R e}\left[\frac{\partial u}{\partial r} n_{r}^{2}+\left(\frac{\partial u}{\partial z}+\frac{\partial v}{\partial r}\right) n_{z} n_{r}+\frac{\partial v}{\partial z}\right) n_{z}^{2}\right]=0, \\
{\left[-2\left(\frac{\partial v}{\partial z}-\frac{\partial u}{\partial r}\right) n_{r} n_{z}+\left(\frac{\partial u}{\partial z}+\frac{\partial v}{\partial r}\right)\left(n_{r}^{2}-n_{z}^{2}\right)\right]=0,}
\end{gathered}
$$

bem como as equações da "shear rate" e da continuidade que são dadas por

$$
q=\sqrt{2\left(\frac{\partial u}{\partial r}\right)^{2}+2\left(\frac{\partial v}{\partial z}\right)^{2}+2\left(\frac{u}{r}\right)^{2}+\left(\frac{\partial u}{\partial z}+\frac{\partial v}{\partial r}\right)^{2}} .
$$


respectivamente.

$$
\frac{1}{r} \frac{\partial(r u)}{\partial r}+\frac{\partial v}{\partial z}=0
$$

Assumindo que o espaçamento da malha é suficientemente pequeno, de forma que a superfície intercepte a célula em dois lados, os seguintes casos são considerados:

(i) Célula surface $(\mathrm{S})$ com apenas um lado em comum com uma célula empty (E): Nessas células é assumido que a superfície é horizontal ou vertical de acordo com qual dos lados é contíguo à célula empty $(\mathrm{E})$. Neste caso temos que o vetor normal à superfície é $\mathbf{n}=\left(n_{r}, 0\right)$ ou $\mathbf{n}=\left(0, n_{z}\right)$.

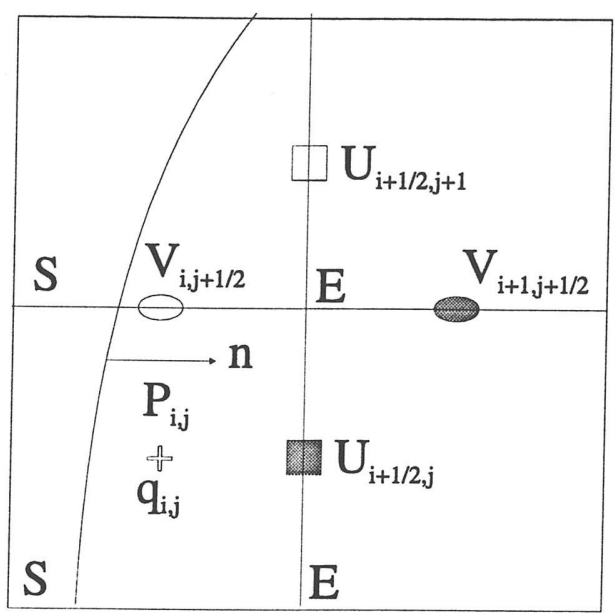

Fig. 4. Célula surface com o lado direito contíguo com uma célula empty.

Consideremos o caso particular de uma célula $\mathrm{S}$ com somente a face direita contígua com uma célula $\mathrm{E}$ conforme mostrado na figura 4 . Nesse caso, $\mathbf{n}=(1,0)$ e introduzindo-o nas equações (4.9) e (4.10) resulta:

$$
\begin{gathered}
\tilde{p}-\frac{2}{R e} \nu(q)\left[\frac{\partial u}{\partial r}\right]=0, \\
\frac{\partial u}{\partial z}+\frac{\partial v}{\partial r}=0,
\end{gathered}
$$

respectivamente. Observamos que quando calculamos as velocidades $\tilde{\mathbf{u}}$ através das equações (4.3) e (4.4), os valores da pressão $\tilde{p}_{i, j}$ e das velocidades $u_{i+1 / 2, j}$ e $v_{i+1, j+1 / 2}$ são requeridos. 
Estes valores podem ser obtidos através das equações (4.13), (4.14) e da equação da continuidade (4.12) como segue:

Primeiramente, discretizando a equação da continuidade no centro da célula fornece:

$$
\frac{1}{r_{i}}\left(\frac{r_{i+1 / 2} u_{i+1 / 2, j}-r_{i-1 / 2} u_{i-1 / 2, j}}{\delta r}\right)+\frac{v_{i, j+1 / 2}-v_{i, j-1 / 2}}{\delta z}=0
$$

donde obtemos

$$
u_{i+1 / 2, j}=\frac{1}{r_{i+1 / 2}}\left(r_{i-1 / 2} u_{i-1 / 2, j}-\frac{r_{i} \delta_{r}}{\delta z}\left(v_{i, j+1 / 2}-v_{i, j-1 / 2}\right)\right) .
$$

Agora discretizando a equação (4.14) na posição $(i+1 / 2, j+1 / 2)$ tem-se:

$$
\frac{u_{i+1 / 2, j+1}-u_{i+1 / 2, j}}{\delta z}+\frac{v_{i+1, j+1 / 2}-v_{i, j+1 / 2}}{\delta r}=0
$$

que fornece

$$
v_{i+1, j+1 / 2}=v_{i, j+1 / 2}-\frac{\delta r}{\delta z}\left(u_{i+1 / 2, j+1}-u_{i+1 / 2, j}\right) .
$$

Tendo calculado as velocidades $u_{i+1 / 2, j}$ e $v_{i+1, j+1 / 2}$, a pressão $\widetilde{p}_{i, j}$ é calculada através da equação (4.13):

$$
\widetilde{p}_{i, j}=\frac{2}{R e} \nu\left(q_{i, j}\right)\left(\frac{u_{i+1 / 2, j}-u_{i-1 / 2, j}}{\delta r}\right) .
$$

O valor de $\nu\left(q_{i, j}\right)$ é obtido como segue: de (4.14) vemos que a "shear rate" (4.11) se reduz a:

$$
q=\sqrt{2\left(\left(\frac{\partial u}{\partial r}\right)^{2}+\left(\frac{\partial v}{\partial z}\right)^{2}+\left(\frac{u}{r}\right)^{2}\right)}
$$

que aplicada no centro da célula produz

$$
q_{i, j}=\left[2\left(\frac{\left(u_{i+1 / 2, j}-u_{i-1 / 2, j}\right)^{2}}{\delta r^{2}}+\frac{\left(v_{i, j+1 / 2}-v_{i, j-1 / 2}\right)^{2}}{\delta z^{2}}+\frac{\left(u_{i+1 / 2, j} u_{i-1 / 2, j}\right)}{r_{i}{ }^{2}}\right)\right]^{\frac{1}{2}} .
$$

Calculado $q_{i, j}$ por (4.15), o valor de $\nu\left(q_{i, j}\right)$ é obtido por

$$
\nu\left(q_{i, j}\right)=\nu_{\infty}+\frac{\nu_{0}-\nu_{\infty}}{\left(1+\left(K q_{i, j}\right)^{m}\right)} .
$$


Para outras configurações de células surface com apenas um lado contíguo com uma célula empty os valores da pressão $\widetilde{p}$ e das velocidades na célula $\mathrm{S}$ e na célula $\mathrm{E}$ são obtidos de maneira análoga.

(ii) Célula surface (S) com dois lados contíguos com células empty (ver fig. 5.). Nesse caso, tomamos $\mathbf{n}=( \pm \sqrt{2} / 2, \pm \sqrt{2} / 2)$ e as condições de contorno na superfície livre conforme as equações (4.9) e (4.10) se reduzem a:

$$
\begin{gathered}
\tilde{p}= \pm \frac{1}{R e} \nu(q)\left[\frac{\partial u}{\partial r}+\frac{\partial v}{\partial z}+\left(\frac{\partial u}{\partial z}+\frac{\partial v}{\partial r}\right)\right] \\
\frac{\partial u}{\partial r}-\frac{\partial v}{\partial z}=0
\end{gathered}
$$

respectivamente.

O sinal em (4.17) é escolhido de acordo com a posição das células E.

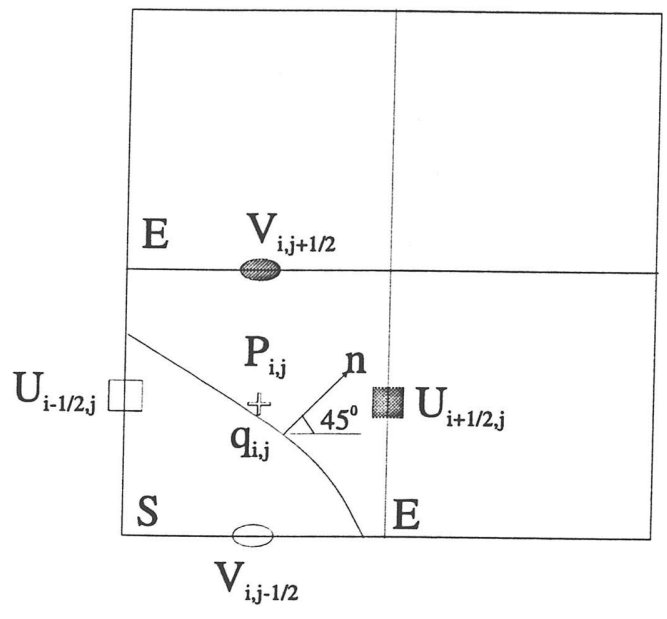

Fig. 5. Células surface com dois lados contíguos com células empty.

Por exemplo, considerando a figura 5 , os valores de $\tilde{p}_{i, j}, u_{i+1 / 2, j}$ e $v_{i, j+1 / 2}$ são requeridos ao se efetuar o cálculo de $\tilde{u}_{i+1 / 2, j}$. As velocidades na face da célula $\mathrm{E}$ são obtidas aplicando a equação da continuidade (4.12) e a equação (4.18) no centro da célula S. Nesse caso temos:

$$
\begin{aligned}
\frac{1}{r_{i}}\left(\frac{r_{i+1 / 2} u_{i+1 / 2, j}-r_{i-1 / 2} u_{i-1 / 2, j}}{\delta r}\right)+\frac{v_{i, j+1 / 2}-v_{i, j-1 / 2}}{\delta z} & =0 \\
\frac{u_{i+1 / 2, j}-u_{i-1 / 2, j}}{\delta r}+\frac{v_{i, j+1 / 2}-v_{i, j-1 / 2}}{\delta z} & =0 .
\end{aligned}
$$


Vemos que as equações (4.19) e (4.20) fornecem um sistema linear $2 \times 2$ para $u_{i+1 / 2, j}$ e $v_{i, j+1 / 2}$. Resolvendo esse sistema obtém-se:

$$
u_{i+\frac{1}{2}, j}=\frac{r_{i}+r_{i-\frac{1}{2}}}{r_{i}+r_{i+\frac{1}{2}}} u_{i-\frac{1}{2}, j} ; \quad v_{i, j+\frac{1}{2}}=v_{i, j-\frac{1}{2}}+\frac{\delta z}{\delta r}\left(u_{i+\frac{1}{2}, j}-u_{i-\frac{1}{2}, j}\right) .
$$

Uma vez calculadas as velocidades $u_{i+1 / 2, j}$ e $v_{i, j+1 / 2}$, o valor de $\widetilde{p}_{i, j}$ é obtido por:

$$
\begin{aligned}
\tilde{p}_{i, j}= & \frac{1}{R e} \nu\left(q_{i, j}\right)\left[\frac{u_{i+1 / 2, j}-u_{i-1 / 2, j}}{\delta r}+\frac{v_{i, j+1 / 2}-v_{i, j-1 / 2}}{\delta z}\right. \\
& +\frac{1}{2}\left(\frac{u_{i+1 / 2, j}+u_{i-1 / 2, j}-u_{i+1 / 2, j-1}-u_{i-1 / 2, j-1}}{\delta z}\right. \\
& \left.\left.+\frac{v_{i, j+1 / 2}+v_{i, j-1 / 2}-v_{i-1, j+1 / 2}-v_{i-1, j-1 / 2}}{\delta r}\right)\right] .
\end{aligned}
$$

O valor de $\nu\left(q_{i, j}\right)$ é obtido por (4.16) onde $q_{i, j}$ é calculado como segue: de (4.18), a equação da "shear rate" (4.11) se reduz a:

$$
q=\sqrt{4\left(\frac{\partial u}{\partial r}\right)^{2}+2\left(\frac{u}{r}\right)^{2}+\left(\frac{\partial u}{\partial z}+\frac{\partial v}{\partial r}\right)^{2}}
$$

que discretizada no centro da célula $S$, fornece a seguinte aproximação:

$$
\begin{aligned}
q_{i, j}= & {\left[4 \frac{\left(u_{i+1 / 2, j}-u_{i-1 / 2, j}\right)^{2}}{\delta r^{2}}+2 \frac{\left(u_{i+1 / 2, j} u_{i-1 / 2, j}\right)}{r_{i}{ }^{2}}\right.} \\
& +\left(\frac{u_{i+1 / 2, j}+u_{i-1 / 2, j}-u_{i+1 / 2, j-1}-u_{i-1 / 2, j-1}}{2 \delta z}\right. \\
& \left.\left.+\frac{v_{i, j+1 / 2}+v_{i, j-1 / 2}-v_{i-1, j+1 / 2}-v_{i-1, j-1 / 2}}{2 \delta r}\right)^{2}\right]^{\frac{1}{2}} .
\end{aligned}
$$

Para outras configurações de células $\mathrm{S}$ com dois lados adjacentes contíguos com células $E$, os valores das velocidades nas faces das células $E$ e a pressão na célula $S$ são obtidos de maneira análoga.

iii) Células $\mathrm{S}$ com dois lados opostos contíguos com células $\mathrm{E}$ ou células S com 3 lados contíguos com células $\mathrm{E}$.

Para essas células não se obtêm uma aproximação para o vetor normal $\mathbf{n}$. Nessas células pelo menos um valor da velocidade é ajustado de modo a satisfazer a equação da continuidade e na pressão é colocado o valor zero. Se essas células aparecerem durante a execucão de um dado problema, a malha deverá ser refinada evitando assim seu aparecimento. 


\subsection{Cálculo do Time-Step}

Um procedimento para otimizar o tamanho do "time-step" em cada ciclo de cálculo é empregado. O "time-step" é calculado segundo as seguintes restrições:

(a) Nenhuma partícula pode cruzar mais do que uma célula em um dado intervalo de tempo, ou seja,

$$
\delta t<\frac{\delta r}{|u|} \quad \text { e } \quad \delta t<\frac{\delta z}{|v|}
$$

(b) Devido à discretização explícita da equação de momento adotamos a seguinte restrição de estabilidade que envolve o número de Reynolds e a viscosidade:

$$
\delta t<\frac{R e}{2 \nu(q)} \frac{\delta r^{2} \delta z^{2}}{\delta r^{2}+\delta z^{2}}
$$

Para satisfazer (4.22) é suficiente que:

$$
\delta t<\frac{\delta r}{\left|U_{\max }\right|} \quad e \quad \delta t<\frac{\delta z}{\left|V_{\max }\right|}
$$

onde $U_{\max }$ e $V_{\max }$ são os máximos valores de $u$ e $v$.

Para a equação (4.23) ser satisfeita, é suficiente que

$$
\delta t<\frac{R e}{2 \nu_{\max }} \frac{\delta r^{2} \delta z^{2}}{\delta r^{2}+\delta z^{2}}
$$

onde $\nu_{\max }=\max \left\{\nu_{i, j}\right\}$.

A implementação dessas condições segue as mesmas normas como implementado no código GENSMAC.

\subsection{A Solução da Equação de Poisson}

A aplicação da equação de Poisson discreta (4.5) sobre a malha resulta em um sistema de equações lineares que pode ser representado por

$$
A \mathrm{X}=\mathrm{D}
$$

onde $A$ é uma matriz $N \times N$, simétrica e definida positiva, $\mathbf{X}$ é o vetor solução de ordem $N$ e D é o vetor divergente $(\nabla \cdot \widetilde{u})$ de ordem $N$, sendo $N$ o número de células Full dentro da malha. Para resolver este sistema é empregado o método dos gradientes conjugados. 


\subsubsection{Implementação do Método dos Gradientes Conjugados}

A montagem da matriz $A$ é feita aplicando a equação (4.8) em cada célula Full da malha. Enumeramos as células Full da esquerda para a direita e de baixo para cima. Cada célula Full representa uma linha dos coeficientes da matriz $A$ e um elemento em $\mathbf{D}$. As condições de fronteira para $\psi$ vistas no Capítulo 3 afetam diretamente a montagem da matriz. A condição $\frac{\partial \psi}{\partial n}=0$ (condição homogênea de Neumann), aplicada sobre uma célula Full que faz fronteira com uma célula Boundary (B) modifica o elemento da diagonal principal enquanto que a condição $\psi=0$ (condição homogênea de Dirichlet) aplicada sobre uma célula Full que faz fronteira com uma célula Surface (S) zera o elemento fora da diagonal que corresponde a célula Surface. Após a montagem da matriz $A$ e do vetor D aplicamos o método dos Gradientes Conjugados sobre o sistema (4.26) sendo o vetor X inicializado como o vetor da função potencial no "time-step" anterior. Tendo em vista a disposição dos elementos da matriz $A$, pode ser vantajoso empregar um pré-condicionador, o qual acelera a convergência do método dos Gradientes Conjugados.

\subsection{Movimento das Partículas}

O fluido é representado por partículas marcadoras que têm como objetivo indicar a posição da superfície livre afim de que a configuração das células surface possam ser determinadas. Suas posições são atualizadas no fim de cada ciclo de cálculo com a finalidade de fornecer a dinâmica do movimento do fluido. As coordenadas dessas partículas são atualizadas através da solução numérica da equação (3.7), pelo método de Euler. Assim, após atualizar o campo de velocidade, as partículas são movidas de acordo com

$$
\begin{aligned}
& r_{p}^{n+1}=r_{p}+u_{p} \delta t^{n+1} \\
& z_{p}^{n+1}=z_{p}+v_{p} \delta t^{n+1}
\end{aligned}
$$

onde $\left(r_{p}, z_{p}\right)$ representa a posição atual da partícula, $\delta t^{n+1}$ é o "time-step" atual empregado e $\left(r_{p}^{n+1}, z_{p}^{n+1}\right)$ é a posição atualizada. As velocidades $u_{p}, v_{p}$ são calculadas usando uma interpolação bilinear envolvendo as quatro velocidades $u$ e $v$ mais próximas. 


\section{Capítulo 5}

\section{Exemplos Numéricos e Aplicações}

Este capítulo apresenta as alterações necessárias no código GENSMAC para que o mesmo possa simular escoamentos axisimétricos não-Newtonianos. Apresentaremos também resultados numéricos mostrando a aplicabilidade desta nova técnica para o tratamento de escoamentos axisimétricos não-Newtonianos, como mostraremos a seguir.

\subsection{Alterações Realizadas no Código GENSMAC}

A inclusão de coordenadas cilíndricas ao código GENSMAC, conforme o algoritmo apresentado no capítulo 3, alterará apenas os passos (i), (ii), (iii) e (iv) (ver processo descrito na Secção (3.3)). Os passos (v), (vi) e (vii) permanecerão os mesmos como aqueles desenvolvidos para escoamento bidimensional. Desta forma para que o código GENSMAC possa simular problemas de escoamentos axisimétricos não-Newtonianos, as seguintes rotinas foram escritas:

- Uma rotina para calcular a pressão $\tilde{p}(r, z, t)$ de acordo com as condições de contorno na superfície livre apresentadas na Secção 4.5,

- Uma rotina para calcular a "shear-rate" no centro das células Full conforme a discretização vista na Secção 4.1.1,

- Uma rotina para calcular as velocidades intermediárias, $\widetilde{u}$ e $\widetilde{v}$ conforme as equações (4.3) e (4.4) desenvolvidas nas Secções 4.1 e 4.2.

As rotinas que calculam a velocidade sobre as faces das células Empty (Secção 4.5), a solução da equação de Poisson discreta (4.8) e a velocidade final (3.4) são as mesmas contidas no código GENSMAC para o tratamento de escoamentos axisimétricos Newtonianos (ver [9]). Também, as equações para o cálculo da velocidade tangente a superfície livre e as condições de contorno na fronteira rígida são manuseadas similarmente as do escoamento bidimensional com superfícies livres implementadas em GENSMAC (ver [7]). 


\subsection{Exemplos Numéricos e Aplicações}

As alterações acima descritas foram incorporadas ao código GENSMAC com o objetivo de investigar escoamentos axisimétricos não-Newtonianos de um fluido incompressível. Para demonstrar a capacidade da técnica desenvolvida neste trabalho, apresentaremos resultados numéricos obtidos em três simulações de escoamentos axisimétricos não-Newtonianos com superfícies livres.

Para visualizarmos os resultados numéricos apresentados a seguir, utilizamos o programa gráfico VisFreeFlow. Este software foi desenvolvido especificamente para fornecer a visualização dos resultados obtidos pelo GENSMAC. Detalhes deste "package" podem ser obtidos em [9].

\subsubsection{Simulação Numérica do Enchimento de Cavidades}

Muitos processos de fabricação envolvem o enchimento de um molde. O fluido, geralmente é não-Newtoniano cuja viscosidade é uma função conhecida da "shear rate". Logo, existe um grande interesse por parte da indústria para prever o comportamento do escoamento durante o estágio de enchimento, afim de evitar resultados indesejáveis (por exemplo, aparecimento de bolhas, enchimento irregular, etc.) que comprometem a qualidade do produto.

Para ilustrar a aplicabilidade desta nova técnica implementada em GENSMAC, capaz de resolver escoamentos complicados, aplicamos o código para simular o enchimento de um molde circular. Neste exemplo, é empregado um injetor circular, do qual o fluido é injetado dentro do molde (ver figura 6a). Assumindo escoamento axisimétrico, o domínio do escoamento é especificado como mostra a figura $6 \mathrm{~b}$.

a

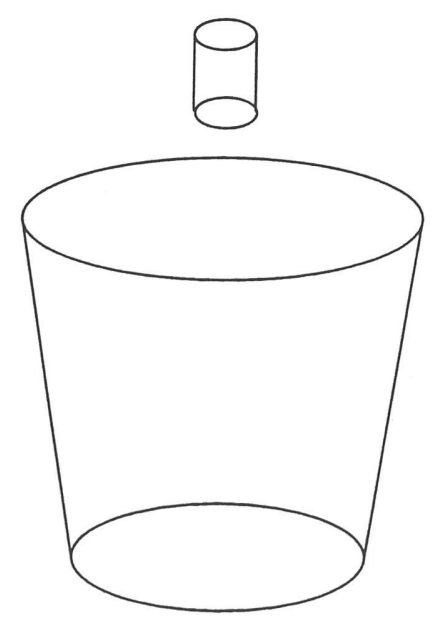

b

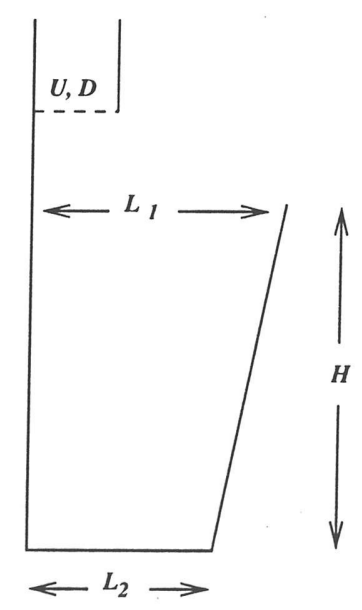

Fig. 6 . Domínio do escoamento em um tubo circular.

Para simular o enchimento do molde, os seguintes parâmetros de entrada foram empregados para executar o código. 
$U=1.0 \mathrm{~ms}^{-1}$ (velocidade do fluido na saída do injetor), $D=10 \mathrm{~mm}$ (diâmetro do injetor).

O fluido é modelado pelo Cross Model, definido por: $\nu_{\infty}=0.001 \mathrm{~m}^{2} \mathrm{~s}^{-1}, \nu_{0}=0.01 \mathrm{~m}^{2} \mathrm{~s}^{-1}$, $K=0.015, m=1.0$. Assim, obtemos um número de Reynolds $R e=U L / \nu_{0}=1.0 \mathrm{e}$ $1 / F^{2}=0.01$. O domínio do escoamento (conforme mostrado na figura $6 \mathrm{~b}$ ) foi definido por $L_{1}=5 \mathrm{~cm}, L_{2}=4 \mathrm{~cm}$ e $H=7 \mathrm{~cm}$. O injetor foi situado a $1 \mathrm{~cm}$ acima do molde. Uma malha de espaçamento $\delta r=\delta z=1 \mathrm{~mm}$, foi empregada $(52 \times 82$ células $)$. Os efeitos térmicos foram desprezados.

Os resultados desta simulação são mostrados na figura 7 a seguir:

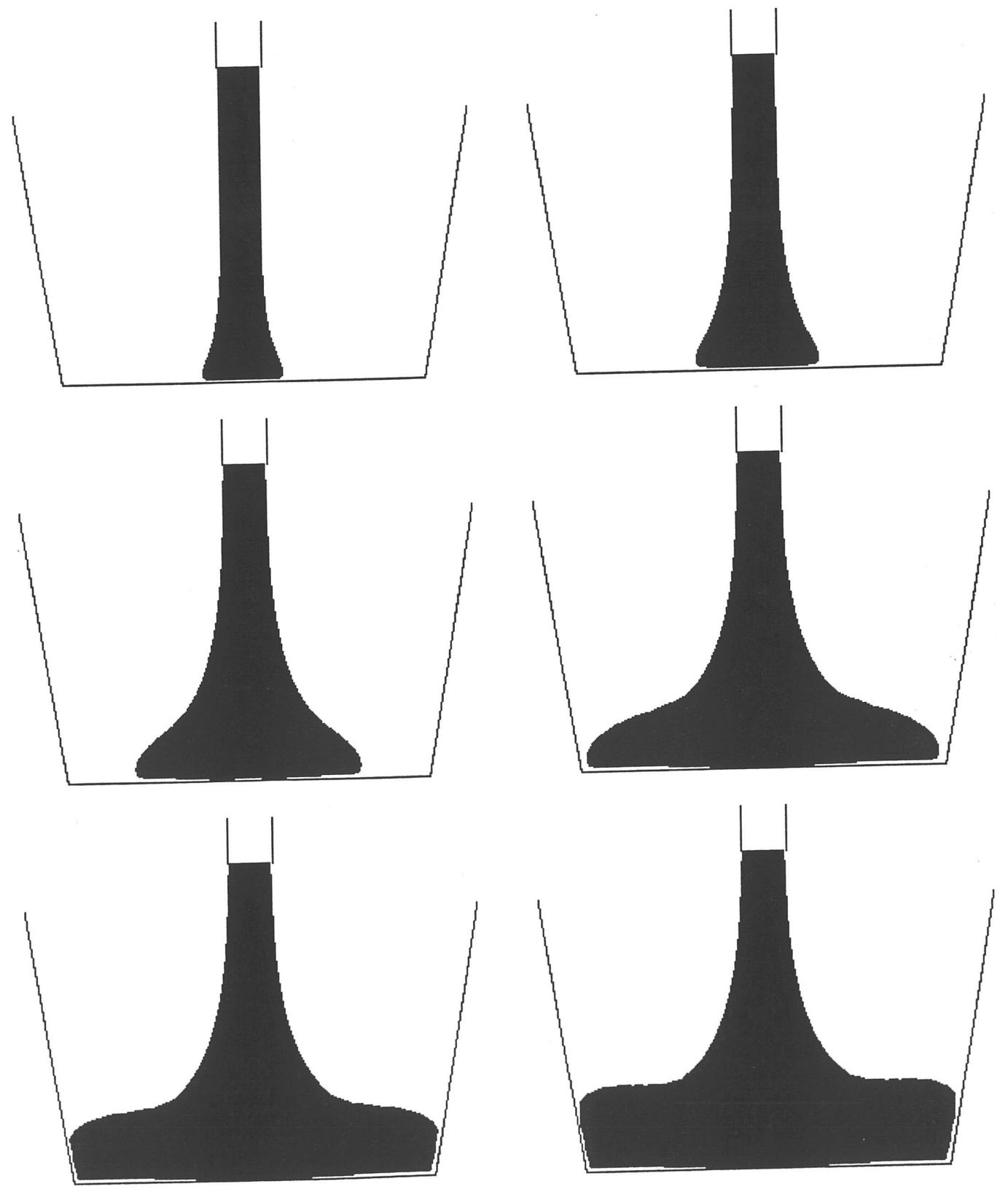

Fig. 7 . Enchimento de um tubo circular em diferentes tempos. 
Para mostrarmos que a técnica apresentada nesse trabalho simula de fato escoamentos não-Newtonianos, apresentaremos a seguir os resultados de três simulações numéricas onde observamos o comportamento não-Newtoniano de um fluido definido pelo Cross Model. Na primeira simulação utilizamos uma viscosidade Newtoniana $\nu=\nu_{0}=0.01$ ( $K=0$ no Cross Model); na segunda simulação utilizamos o Cross Model definido por $\nu_{\infty}=0.001, \nu_{0}=0.01, K=0.15 m=1.0$ e na terceira simulação foi utilizada uma viscosidade Newtoniana $\nu=\nu_{\infty}=0.001$. Os resultados dessas simulações são mostrados na figura 8 , onde as fotos (figuras) da coluna da esquerda ilustram o comportamento do fluido Newtoniano de $\nu=\nu_{0}$ em diferentes tempos, a coluna do meio e a coluna da esquerda ilustram os fluidos não- Newtoniano e Newtoniano de $\nu=\nu_{\infty}$, respectivamente.

a

b

c
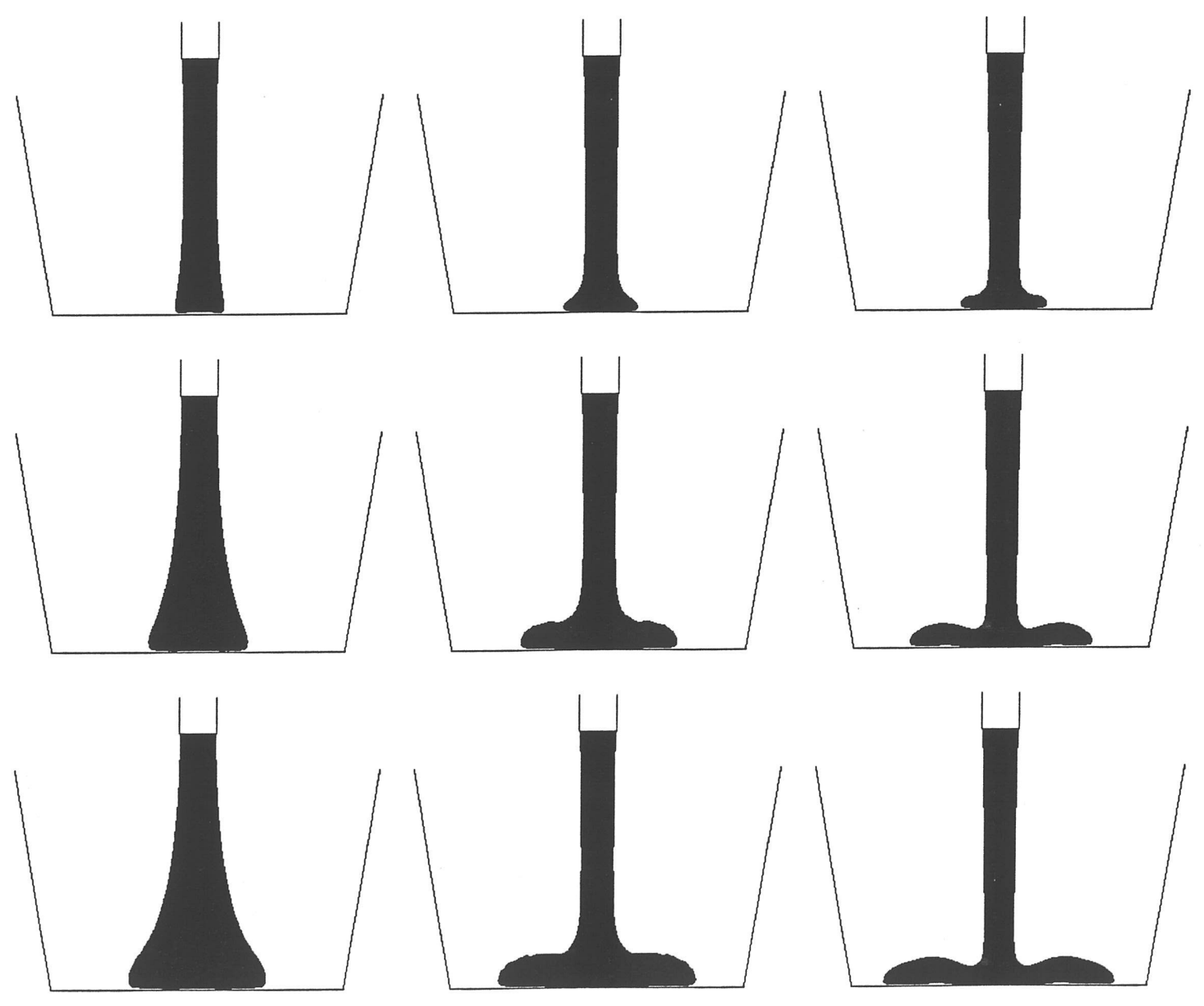

Fig. 8. Enchimento de um tubo circular para diferentes valores de tempo: (a) $\nu=\nu_{0}$ e $\mathrm{K}=0$ (viscosidade Newtoniana), (b) $\nu_{\infty}=0.001, \nu_{0}=0.01$ e $\mathrm{K}=0.15$ (modelo Cross Model, fluido não-Newtoniano), (c) $\nu=\nu_{\infty}$ e $K \approx \infty$ (viscosidade Newtoniana). 

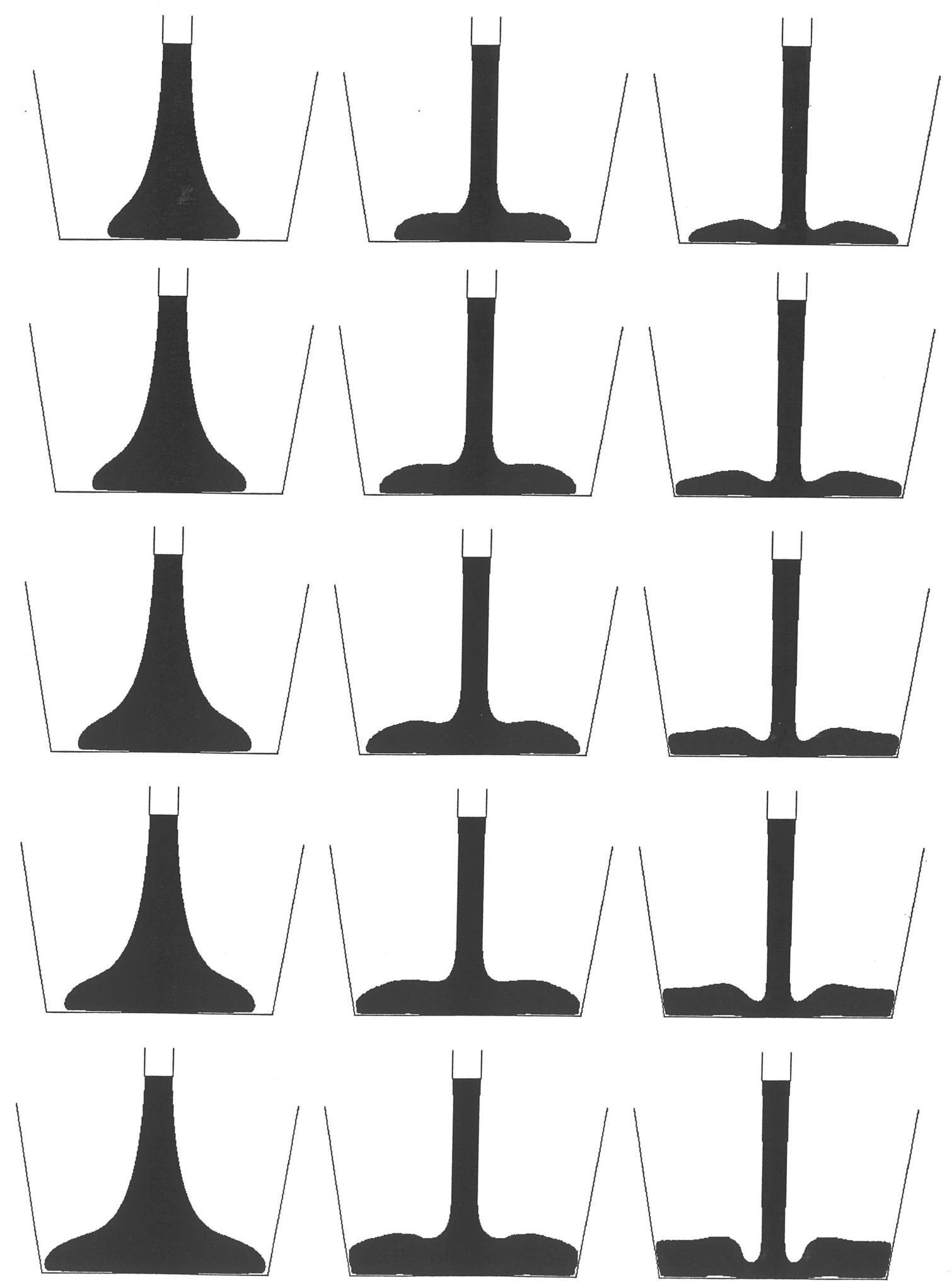

Fig. 8. Continuação. 
Podemos observar na figura 8, que o comportamento do escoamento de um fluido nãoNewtoniano modelado pelo Cross Model é intermediário entre os dois patamares Newtonianos de $\nu=\nu_{0}$ e $\nu=\nu_{\infty}$. Como podemos observar no Cross Model, conforme o valor de $K$ o comportamento do escoamento tende a se aproximar ao comportamento de um fluido Newtoniano de viscosidade $\nu=\nu_{0}$ ou $\nu_{\infty}$. A seguir, apresentaremos resultados de quatro simulações com o Cross Model utilizado no exemplo da figura 8.

Estas simulações foram realizadas para valores de $K=0.015,0.1$ e 0.5 . A figura 9 mostra uma comparação dos fluidos não-Newtonianos para os valores de $K$ citados acima com o fluido Newtoniano $\left(\nu=\nu_{\infty}\right)$ no mesmo instante $t=18.750$. Note que quanto maior o valor de $K$, o comportamento do fluido não-Newtoniano mais se aproxima ao comportamento do fluido Newtoniano $\operatorname{com} \nu=\nu_{\infty}$. Isso mostra que a técnica apresentada neste trabalho é de fato capaz de simular escoamentos não-Newtonianos.

a

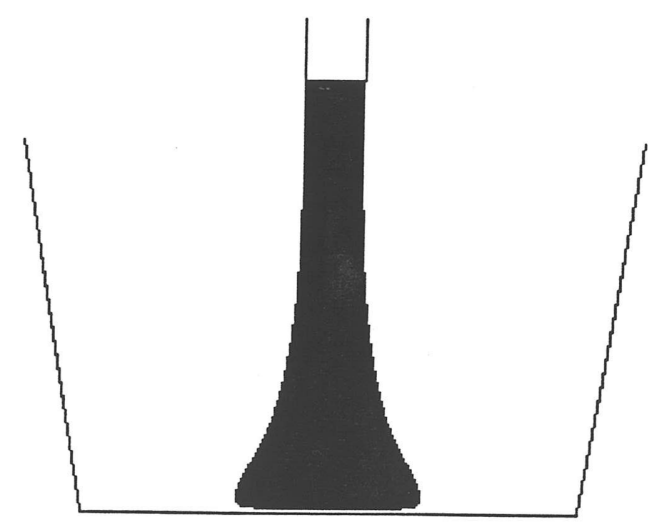

c

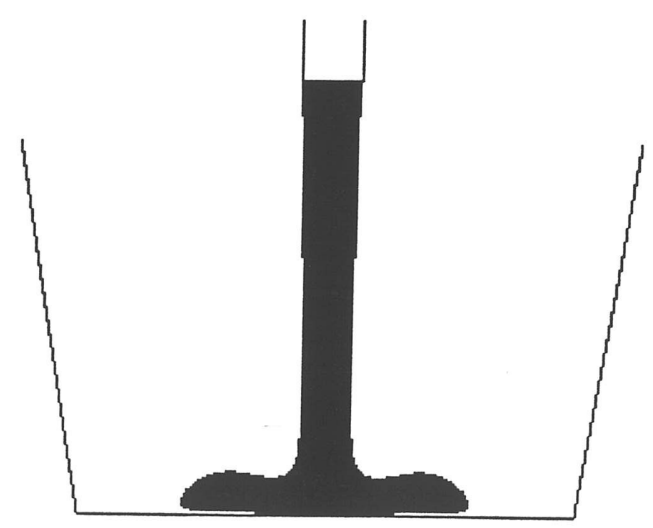

b

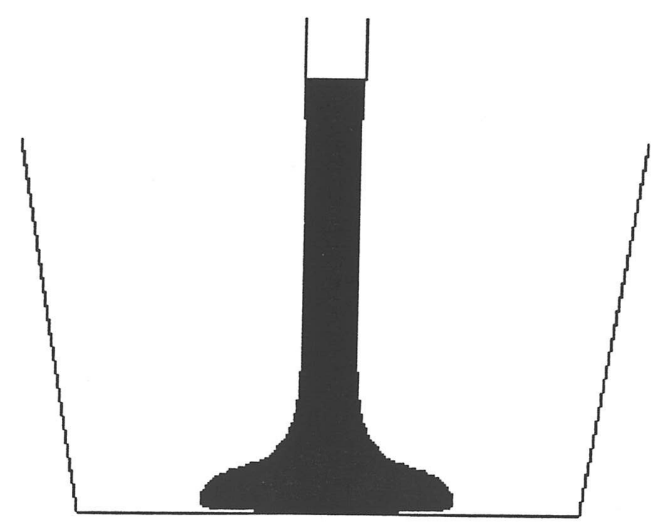

d

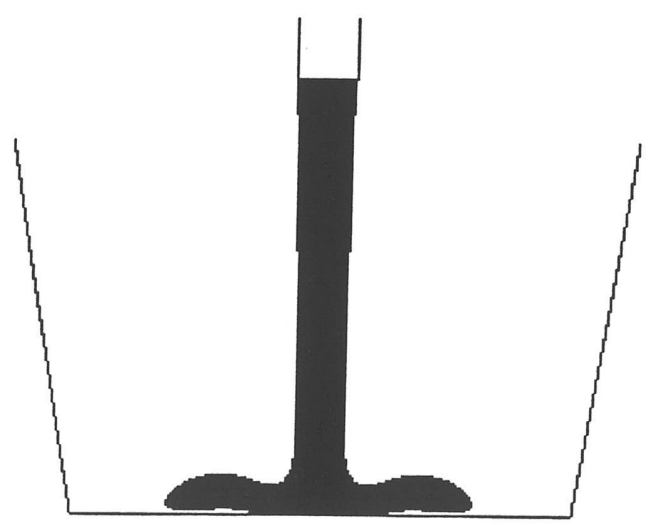

Fig. 9. Enchimento de um tubo circular com fluido Não-Newtoniano no tempo $t=18.750$ para diferentes valores de $K$ : (a) $K=0.015$, (b) $K=0.1$, (c) $K=0.5$ e (d) fluido Newtoniano $\operatorname{com} \nu=\nu_{\infty}$. 


\subsubsection{Simulação Numérica do "Die Swell" de um Jato Axisimé- trico}

Este problema tem atraído a atenção de muitos pesquisadores e várias técnicas para simular o escoamento de um jato emergindo de um tubo tem sido empregadas. Nesse problema podemos ver o fenômeno conhecido como "extrudate swell" ou "jet swell" de um jato saindo de um tubo circular. Esse problema aparece em muitas aplicações industriais, tornando-se assim de interesse industrial compreender o comportamento do "fluid-swelling" para estas aplicações. Devido a complexidade do domínio do escoamento, a técnica de elementos finitos e de diferenças finitas tem sido bem empregadas.

Consideremos um jato circular escoando dentro de um tubo e então espelido para a atmosfera. Para simular esse problema, o domínio computacional e as condições de contorno são mostrados conforme figura 10a. No "inflow" o fluido é introduzido no tubo com velocidade constante $u=U_{I}$ e $v=0$. Devido a condição "no-slip" impostas nas paredes do tubo, a velocidade rapidamente desenvolve um perfil parabólico e na saída do tubo acontece sob certas condições, o fenômeno da expansão (ou contração) do jato. Para simular esse problema assumimos que o escoamento é axisimétrico e utilizamos o domínio computacional conforme mostrado na figura 10b onde no eixo de simetria, a condição "free-slip" é imposta.
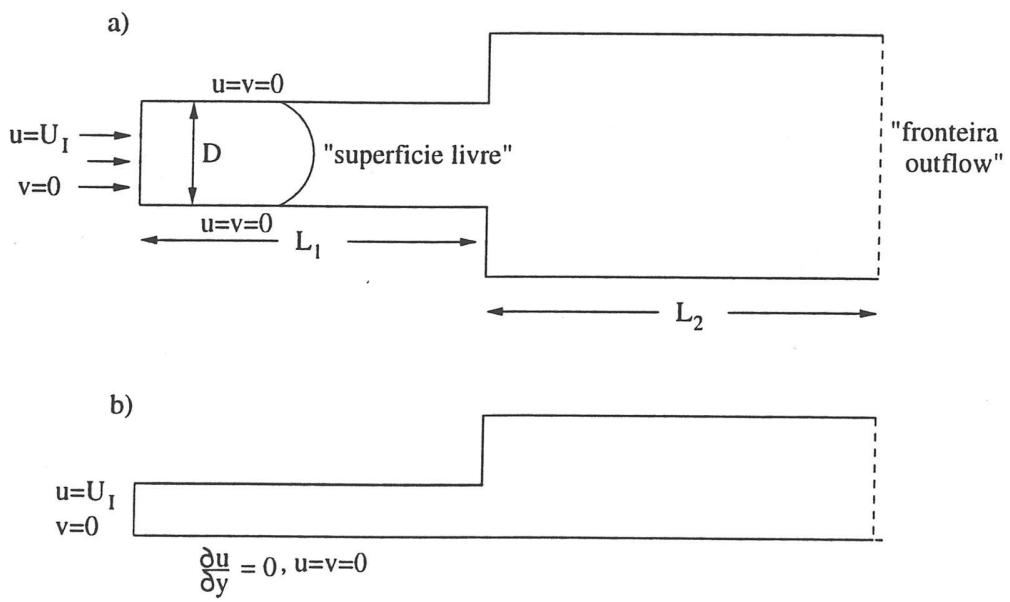

Fig.10. Simulação do "Die Swell": domínio e condições de contorno utilizadas.

Nessa simulação os seguintes parâmetros foram utilizados:

$U_{I}=5 \mathrm{~ms}^{-1}, \quad D=1 \mathrm{~cm}$ (diâmetro do tubo), $L_{1}=3 \mathrm{~cm}$ (comprimento do tubo) e $L_{2}=6 \mathrm{~cm}$ (distância da saída do tubo até o outflow).

A viscosidade foi modelada pelo Cross Model

$$
\nu=\nu_{\infty}+\frac{\nu_{0}-\nu_{\infty}}{\left(1+(K q)^{m}\right)}
$$

onde $\nu_{\infty}=0.001 \mathrm{~m}^{2} \mathrm{~S}^{-1}, \nu_{0}=0.050 \mathrm{~m}^{2} \mathrm{~s}^{-1}$ e $m=1.0$; a gravidade foi desprezada. 
Para observamos o comportamento Não-Newtoniano foram tomados os seguintes valores para a constante $K ; K=0$ (fluido Newtoniano $\operatorname{com} \nu=\nu_{0}$ ), $0.005,0.010$ e 0.015 . $\mathrm{Na}$ figura 11 é mostrado a configuração do jato no instante $t=6.25$ para os diferentes valores de $K$.

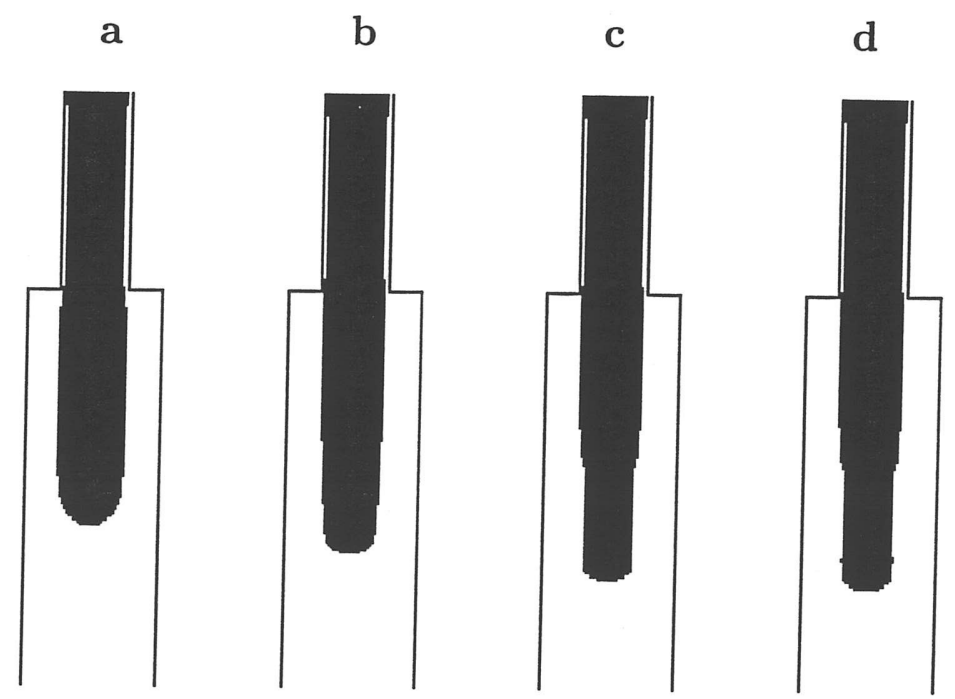

Fig. 11. Die swell de jatos Newtoniano e não-Newtoniano em $t=6.25$ para diferentes valores de $K$ : (a) $K=0$, (b) $K=0.005$, (c) $K=0.010$, (d) $K=0.015$.

Como podemos observar na figura 11, o comportamento não-Newtoniano do fluido está diretamento ligado aos valores de $K$. Observemos que o "swelling" (porção de dilatação) é razoavelmente nítida no caso Newtoniano, diminui com o aumento do valor de $K$ e o jato é correspondentemente mais longo (os volumes de fluido na figura 11 devem ser iguais, uma vez que o fluido é incompressível). Podemos observar também que quanto maior o valor de $K$ o jato desenvolve, na extremidade, uma região de formato mais alongado e retangular. Esse mesmo efeito foi observado na simulação do "die-swell" de um jato bidimensional apresentado por Tomé et al [6]. Nessa simulação, demonstramos mais uma vez que essa técnica é de fato capaz de simular escoamentos não-Newtonianos. 


\subsubsection{Simulação Numérica do Splashing Drop}

Para demonstrarmos a capacidade do código em resolver escoamentos complexos e verificarmos a precisão das aproximações para as condições de contorno na superfície livre vistas na Seção 4.5, apresentaremos a simulação do "Splashing Drop" de um fluido nãoNewtoniano. Para isso, consideremos uma gota esférica de um fluido caindo numa caixa circular contendo o mesmo fluido (ver figura 12a).

Assumindo escoamento axisimétrico, utilizamos o domínio computacional conforme mostrado na figura $12 \mathrm{~b}$.
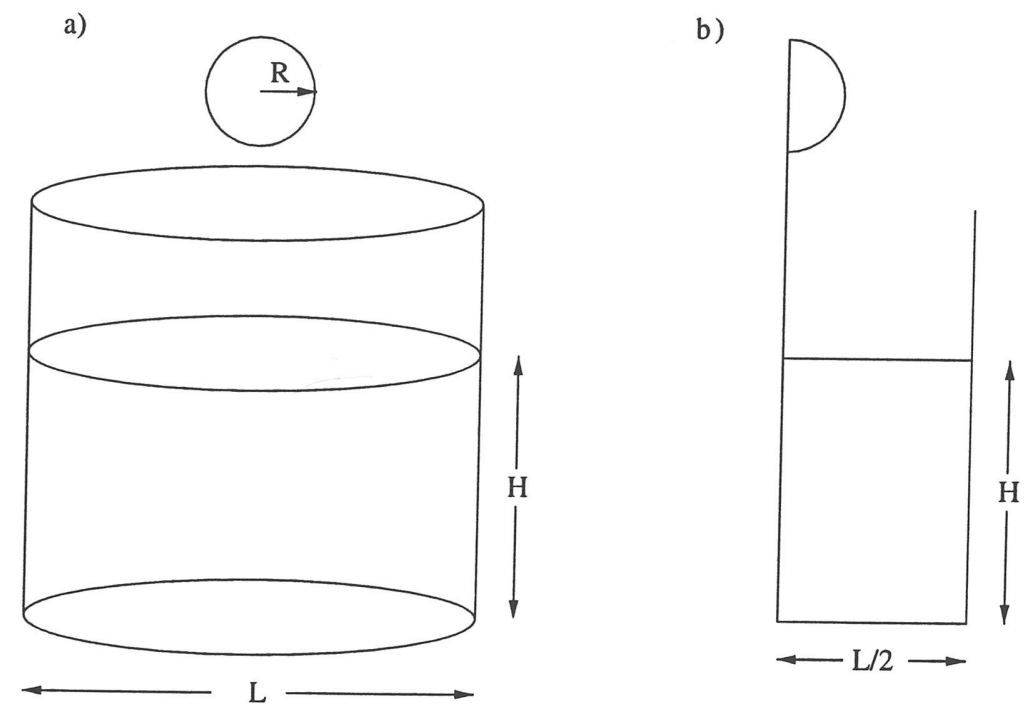

Fig. 12. Simulação do "Splashing Drop": domínio computacional.

Nessa simulação os seguintes parâmetros foram empregados:

$U=1 \mathrm{~ms}^{-1}$ (velocidade inicial da gota),

$R=1.6 \mathrm{~cm}$ (raio da gota), portanto o diâmetro da gota é $D=3.2 \mathrm{~cm}$,

$L=22 \mathrm{~cm}$ (diametro da caixa circular),

$H=7 \mathrm{~cm}$ (altura de fluido na caixa).

O tamanho da célula é $\delta r=\delta z=0.2 \mathrm{~cm}$. A gota situa-se a uma distância de $3 \mathrm{~cm}$ acima da caixa e cai sob a força da gravidade com $g=-981.0 \mathrm{cms}^{-2}$. O fluido é modelado pelo Cross Model onde utilizamos os seguintes valores para as constantes:

$\nu_{\infty}=3.0 \mathrm{~cm}^{2} \mathrm{~s}^{-1}, \nu_{0}=8.0 \mathrm{~cm}^{2} \mathrm{~s}^{-1}, K=0.2$ e $m=1.0$, obtendo assim um número de Reynolds de $R e=U D / \nu_{0}=40$.

A figura 13 mostra o comportamento dos fluido Newtoniano de $\nu=\nu_{0}$ (coluna da esquerda), não-Newtoniano modelado pelo Cross Model (coluna do meio) e fluido Newtoniano de $\nu=\nu_{\infty}$ (coluna da direita) em diferentes tempos. 

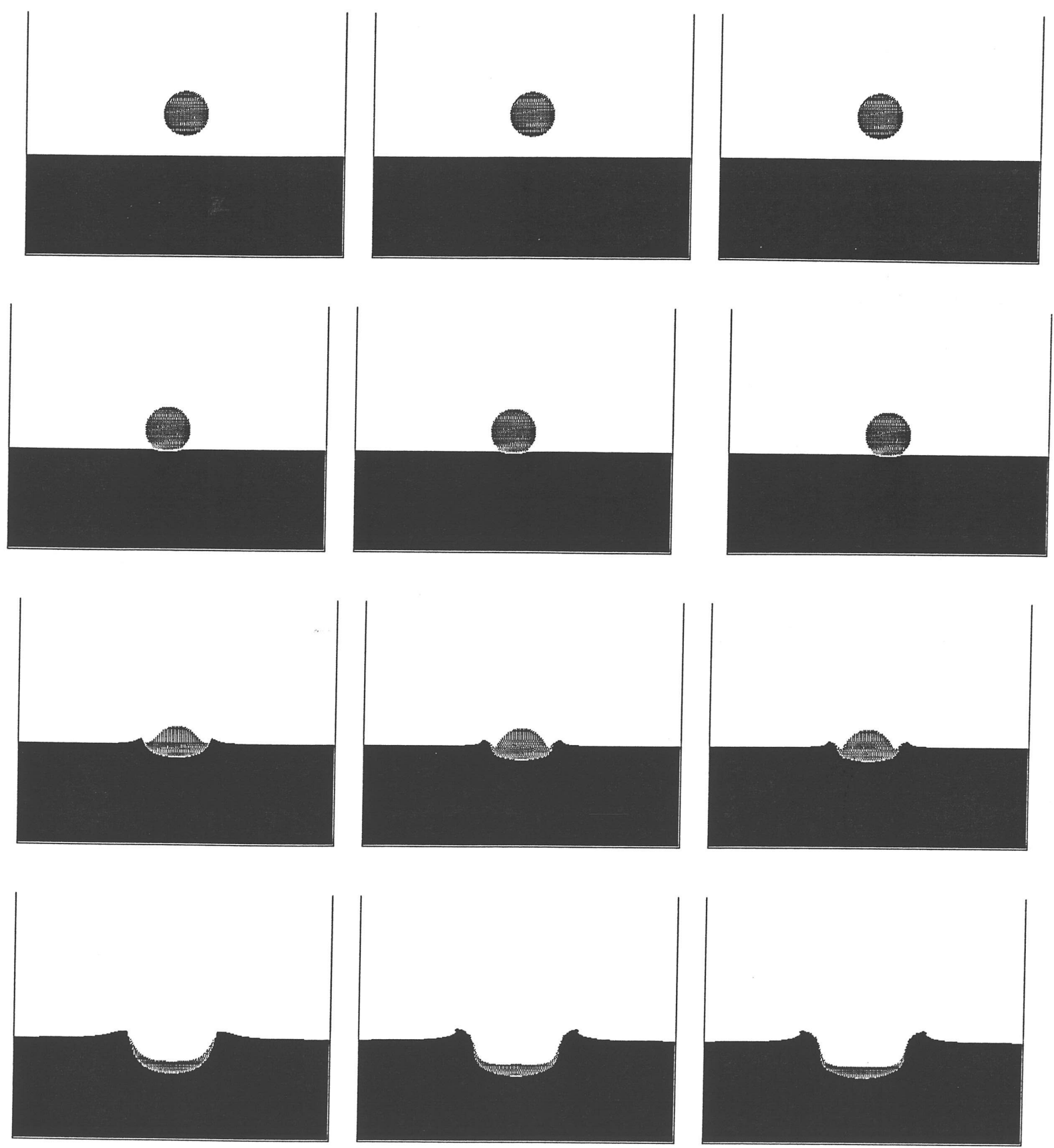

Fig. 13. Comparação entre a simulação do "Splasing Drop" para diferentes valores de tempo: (a) $\nu=\nu_{0}\left(K=0\right.$, viscosidade Newtoniana), (b) $\nu_{\infty}=3.0, \nu_{0}=8.0 \mathrm{e}$ $K=0.2$ (modelo Cross Model, fluido não-Newtoniano), (c) $\nu=\nu_{\infty}(K \approx \infty$ viscosidade Newtoniana). 

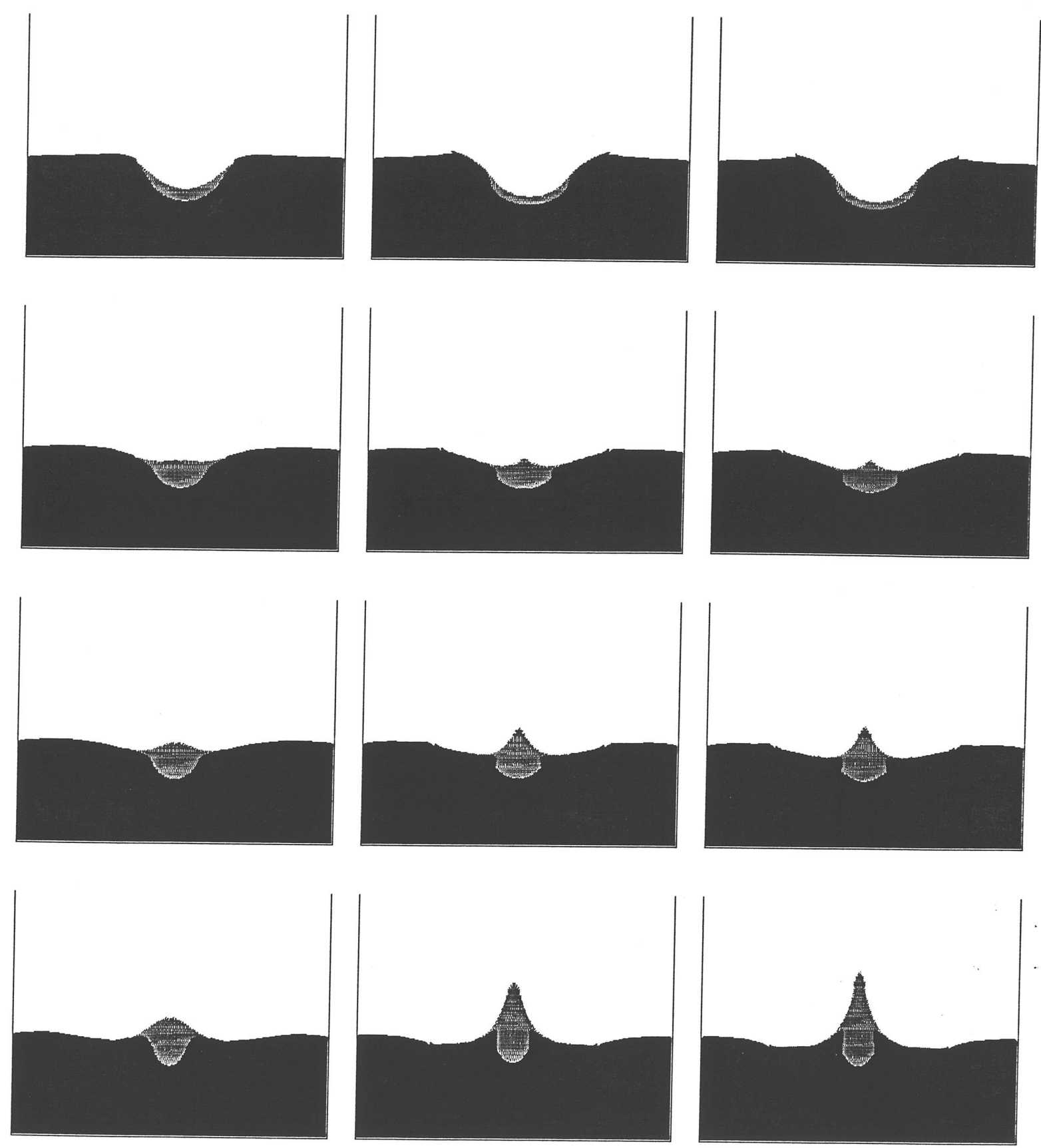

Fig. 13. Continuação. 

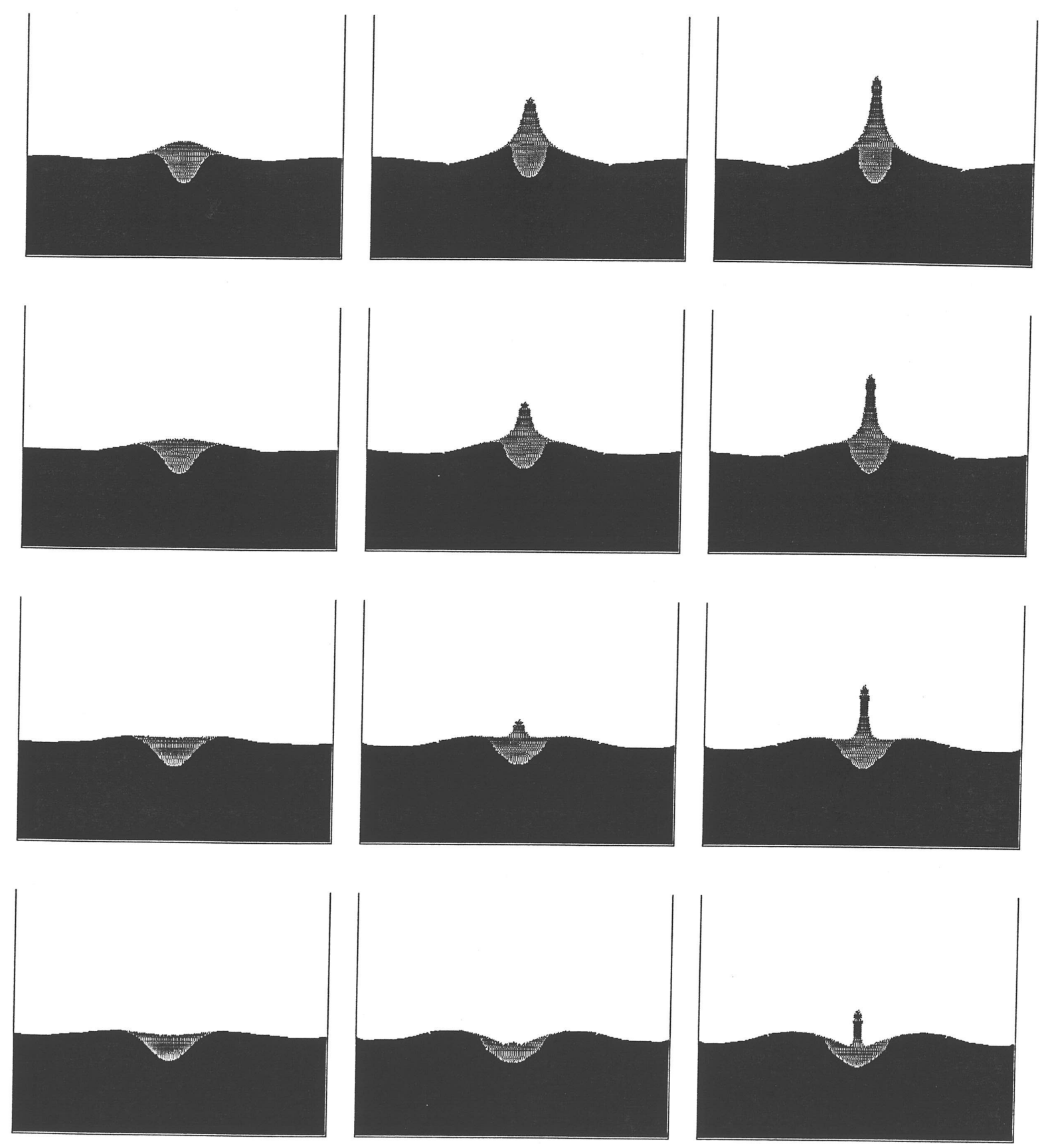

Fig. 13. Continuação. 
Como foi possível observar, o comportamento do escoamento de um fluido não-Newtoniano modelado pelo Cross Model é intermediário entre os dois patamares Newtonianos de $\nu=\nu_{0}$ e $\nu=\nu_{\infty}$. Esta simulação foi utilizada para validar o método apresentado neste trabalho e comprovarmos mais uma vez que esta técnica realmente simula escoamentos não-Newtonianos.

Para melhor visualização do exemplo "Splashing Drop", apresentamos aqui os resultados mostrados na figura 13 em três dimensões.
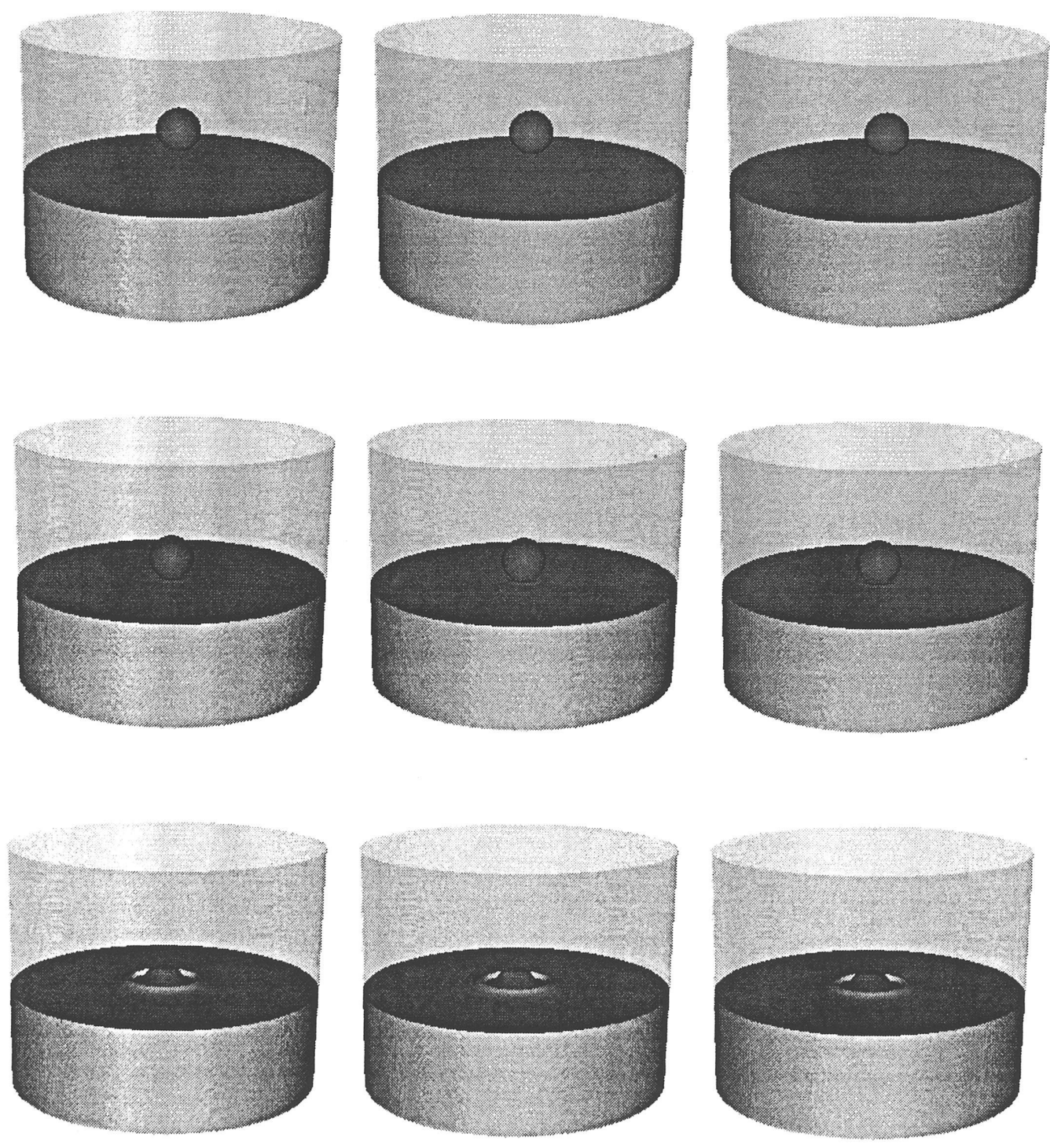

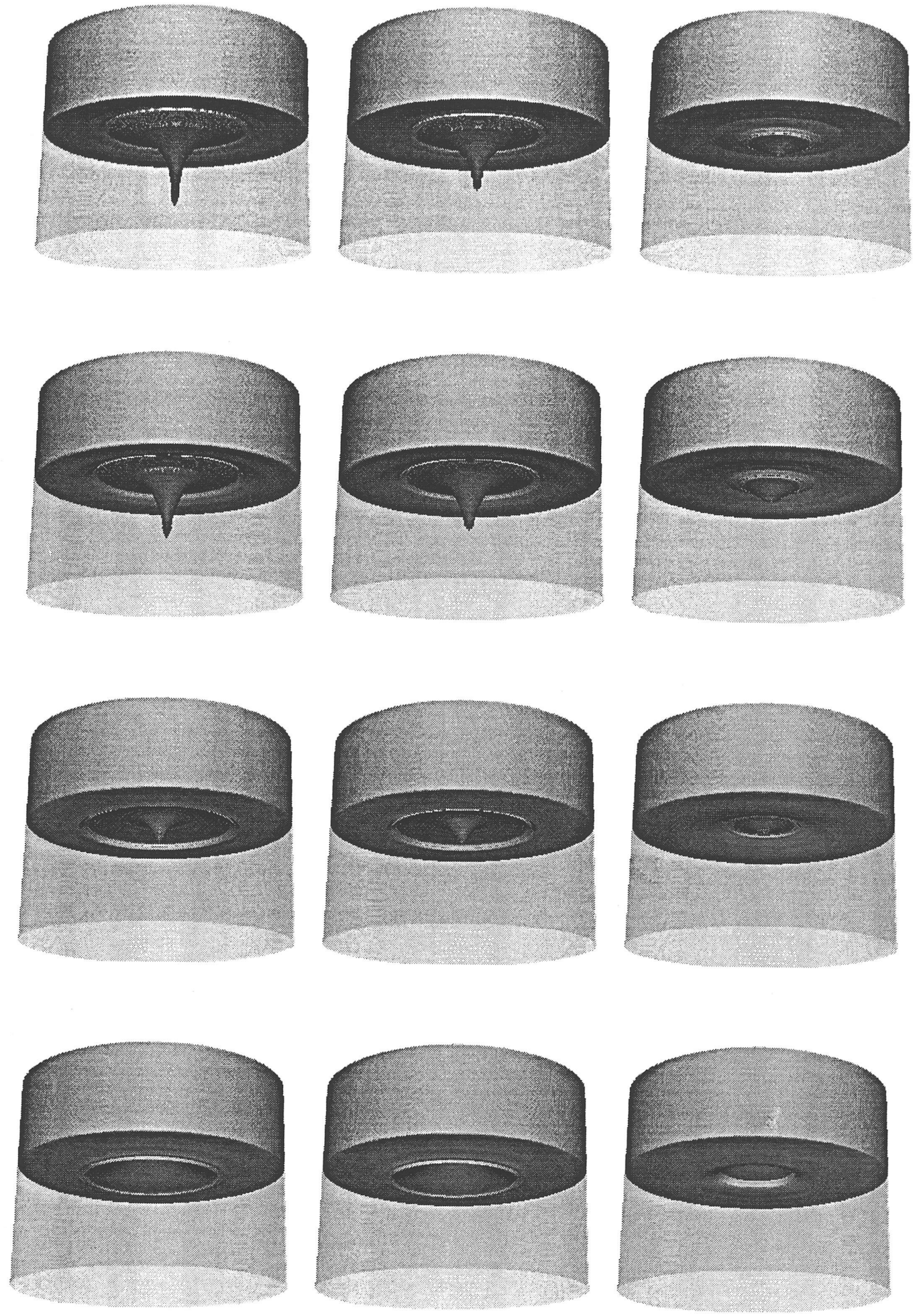


\section{Capítulo 6}

\section{Resumo e Conclusões}

Neste trabalho desenvolvemos um método numérico para resolver escoamentos axisimétricos de fluidos não-Newtoniano. Trata-se de uma extensão do código GENSMAC [7], um programa escrito na linguagem FORTRAN 77 utilizando programação estruturada.

Até o momento o código tratava apenas de escoamentos não-Newtonianos bidimensionais. Com a inclusão de coordenadas cilíndricas tornou-se necessário que algumas alterações fossem incorporadas ao código, que possibilitaram a investigação de escoamentos axisimétricos não-Newtonianos de um fluido incompressível, aumentando a gama de aplicabilidade do GENSMAC. O método apresentado neste trabalho tem obtido bons resultados no tratamento de problemas que ainda não haviam sido tratados com o código existente. Desta forma o código torna-se uma ferramenta útil para engenharia e indústrias.

Esta dissertação foi dividida em 3 partes principais:

- A primeira parte é constituída dos capítulos 2 e 3 :

No capítulo 2 foi feito um estudo das equações fundamentais para escoamentos viscosos incompressíveis com superfícies livres para um fluido não-Newtoniano. Desenvolvemos as equações de momento, de massa, da shear rate e das condições de contorno na superfície livre em coordenadas cilíndricas.

No capítulo 3 apresentamos em linhas gerais o código Gensmac, mostrando algumas características fundamentais juntamente com o algoritmo computacional. Foi vista também a aplicação das condições de fronteira "no-slip" e "free-slip" para obter-se uma aproximação para a "shear rate".

- A segunda parte contitui-se do quarto capítulo:

Neste capítulo apresentamos um estudo realizado com a técnica de diferenças finitas. Apresentamos uma aproximação para o cálculo da velocidade $\tilde{\mathbf{u}}(r, z, t)$, uma 
aproximação para equação de Poisson juntamente com sua resolução pelo método dos gradientes conjugados.

- A última parte compreende o quinto capítulo:

Aqui foram discutidas as alterações realizadas no código GENSMAC com o objetivo de generalizá-lo para resolver escoamentos axisimétricos não-Newtonianos com superfícies livres. Para ilustrar melhor a aplicabilidade desta nova técnica implementada em GENSMAC, o código foi empregado para resolver três problemas que envolvem superfícies livres: enchimento de moldes, "die swell" de um jato circular e a simulação do "splashing drop". O primeiro trata-se de problemas como o enchimento de um molde circular, o segundo é um estudo a respeito do "extrudate swell" de um tubo circular onde é possível observar claramente o efeito não-Newtoniano. Finalmente, o terceiro trata-se de um exemplo clássico da dinâmica dos fluidos que foi utilizado aqui para "validar" os resultados numéricos desta nova técnica.

Como continuidade do trabalho apresentado nessa dissertação, propomos os seguintes tópicos:

- Implementação de um pré-condicionador para a equação de Poisson,

- Implementação de um método implícito para calcular as tilde-velocidades,

- Inclusão da temperatura no método,

- Inclusão de tensão superficial,

- Extensão do modelo para fluidos visco-elásticos do tipo Maxwell e fluidos de segunda ordem. 


\section{Referências Bibliográficas}

[1] Harlow, F. and Welsh, J. E.;1965, "Numerical Calculation of Time-Dependent Viscous Incompressible Flow of Fluid with a Free Surface"; Phisics of Fluids, Vol.8, pp 2182-2193.

[2] Amsden, A.A. and Harlow, F. H., 1971, "The SMAC Method: A numerical Technique for Calculating Incompressible Fluid Flow", Los Alamos scientific Lab., Report LA4370, Los Alamos, New Mexico.

[3] Bachelor, G. K., 1967, An introduction to Fluid Dynamics, (Cambridge University Press, Cambridge).

[4] Fletcher, C. A. J., 1991, Computational Techniques for Fluid Dynamics, (SpringerVerlag Berlin Heidelberg), Vol. I and Vol. II.

[5] Smith, G. D., 1978, Numerical Solution of Partial Differential Equations: Finite Difference Methods, (Oxford University Press, Oxford).

[6] Tomé, M. F., 1993, GENSMAC: A Multiple Free Surface Fluid Flow Solver, Ph.D. Thesis, University of Strthclyde, Glasgow, U. K.

[7] Tomé, M. F. and McKee, S., 1994, "GENSMAC: A Computational Marker-andCell Method for Free Surface Flows in General Domains", Journal of Computational Physics, Vol. 110, pp. 171-189.

[8] Tomé, M. F., Duffy, B. and McKee, S., 1996, "A Numerical Technique for Solving Unsteady non-Newtonian Free Surface Fluid Flow", Journal of Non-Newtonian Fluid Mechanics, Vol. 62, pp. 9-34.

[9] Tomé, M. F., Castelo, A.Fo., Cuminato, J. A. e outros , 1996, "Numerical Simulation of Axysimmetric Free Surface Flows", Relatório Técnico, ICMSC - USP, São Carlos.

[10] Acheson, D. J., 1990, Elementary Fluid Dynamics, (Oxford University Press, Oxford). 